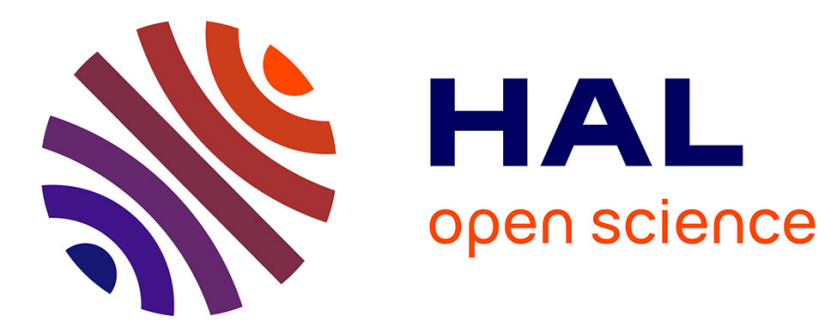

\title{
Bayesian Sequential Testing with Expectation Constraints
}

\author{
Stefan Ankirchner, Maike Klein
}

\section{To cite this version:}

Stefan Ankirchner, Maike Klein. Bayesian Sequential Testing with Expectation Constraints. 2018. hal-01908132

\section{HAL Id: hal-01908132 \\ https://hal.science/hal-01908132}

Preprint submitted on 29 Oct 2018

HAL is a multi-disciplinary open access archive for the deposit and dissemination of scientific research documents, whether they are published or not. The documents may come from teaching and research institutions in France or abroad, or from public or private research centers.
L'archive ouverte pluridisciplinaire HAL, est destinée au dépôt et à la diffusion de documents scientifiques de niveau recherche, publiés ou non, émanant des établissements d'enseignement et de recherche français ou étrangers, des laboratoires publics ou privés. 


\title{
Bayesian Sequential Testing with Expectation Constraints
}

\author{
Stefan Ankirchner * $\quad$ Maike Klein ${ }^{\dagger}$
}

October 29, 2018

\begin{abstract}
We study a stopping problem arising from a sequential testing of two simple hypotheses $H_{0}$ and $H_{1}$ on the drift rate of a Brownian motion. We impose an expectation constraint on the stopping rules allowed and show that an optimal stopping rule satisfying the constraint can be found among the rules of the following type: stop if the posterior probability for $H_{1}$ attains a given lower or upper barrier; or stop if the posterior probability comes back to a fixed intermediate point after a sufficiently large excursion. Stopping at the intermediate point means that the testing is abandoned without accepting $H_{0}$ or $H_{1}$. In contrast to the unconstrained case, optimal stopping rules, in general, cannot be found among interval exit times. Thus, optimal stopping rules in the constrained case qualitatively differ from optimal rules in the unconstrained case.
\end{abstract}

$2010 M S C:$ 62L10, 60G40, 62L15.

Keywords : Bayesian sequential testing, optimal stopping, expectation constraint.

\section{Introduction}

Let $X$ be a Brownian motion with either drift rate 0 or drift rate $\kappa \neq 0$. Suppose that an agent observes the process $X$ and aims at finding a time when to stop the observation and then to accept one of the two hypotheses

$$
H_{0}: \text { drift rate is } 0, \quad H_{1}: \text { drift rate is } \kappa .
$$

More precisely, let $Y_{0}$ be the a priori probability the agent assigns to the event that $H_{1}$ is true. For $t>0$ let $Y_{t}$ be the posterior probability of $H_{1}$ to be true given the observation of $X$ on $[0, t]$. Let $\left(\mathcal{F}_{t}^{Y}\right)$ be the filtration generated by $\left(Y_{t}\right)$ and denote by $\mathcal{T}(T)$ the set of all $\left(\mathcal{F}_{t}^{Y}\right)$-stopping times satisfying the constraint $\mathbb{E}[\tau] \leq T \in[0, \infty)$. Let $\alpha \in\left(0, \frac{1}{2}\right), \beta \geq 1$ and suppose that the agent faces the stopping problem

$$
\operatorname{maximize} \mathbb{E}\left[\mathbb{1}_{(0, \alpha]}\left(Y_{\tau}\right)+\beta \mathbb{1}_{[1-\alpha, 1)}\left(Y_{\tau}\right)\right] \quad \text { subject to } \tau \in \mathcal{T}(T)
$$

In this article we solve the stopping problem (0.1). The problem gathers in a stylized form a situation where an agent continuously collects data with the aim to ultimately decide between two hypotheses. For example, a pharmaceutical company performs tests in order to check whether a medicament is clinically effective or not. Another example is a company collecting data on customer preferences in order to decide whether to offer a product or not.

The payoff function in (0.1) reflects that the agent can only accept $H_{0}$ or $H_{1}$ if the posterior probability is below $\alpha$ or above $1-\alpha$, respectively. One can interpret $\alpha$ and $1-\alpha$ as thresholds

${ }^{*}$ Stefan Ankirchner, Institute for Mathematics, University of Jena, Ernst-Abbe-Platz 2, 07743 Jena, Germany. Email: s.ankirchner@uni-jena.de, Phone: +49 (0)3641 946275.

${ }^{\dagger}$ Maike Klein, TU Wien, Institute of Statistics and Mathematical Methods in Economics, Wiedner Hauptstr. 8 / E105-1 \& -5 FAM, 1040 Wien, Austria. Email: maike.klein@fam.tuwien.ac.at, Phone: +43 1 58801105170 .

M.K. was partially supported by the Austrian Science Fund (FWF) under grant P30864. 
predetermined by a regulator or by a company policy. If $\beta>1$, then the agent assigns a higher value to accepting $H_{1}$ than to accepting $H_{0}$.

We are mainly interested in the case where the first hitting time of the thresholds $\alpha$ and $1-\alpha$ has an expectation larger than $T$, the bound on the expectation of any stopping time. In this case, the agent has to choose a stopping time $\tau$ for which the payoff is zero with a positive probability. In other words, the agent has to choose a stopping rule that allows to abandon the observation without accepting $H_{0}$ or $H_{1}$. Such stopping rules can justify to surrender long-running observations or test series even if no significant result has been obtained.

The results from [1] on general stopping problems with expectation constraints imply that for solving (0.1) it is sufficient to consider only stopping times such that the law of the posterior probability process $Y$ at the stopping time is a weighted sum of at most 3 Dirac measures (cf. Theorem 1.4 in [1]). We show that a reduction to 2 mass points is not possible in the specific stopping problem (0.1).

Furthermore, we construct explicit optimal stopping times that are compositions of two consecutive interval exit times. More precisely, we show that if $T$ is sufficiently small and sufficiently large, then there is an optimal stopping rule of the following type: stop if the posterior probability for $H_{1}$ attains $\alpha$ or $1-\alpha$; or stop if the posterior probability comes back to an intermediate point $b^{*}$ after a sufficiently large excursion. In this case, three possible decision outcomes occur with positive probability: $H_{0}$ or $H_{1}$ or none of both is accepted. It turns out that the intermediate point $b^{*}$ does not depend on the time constraint $T$ (only its probability weight does). Consequently, the same 3 points suffice for describing optimal stopping rules.

We show that, in general, there does not exist an optimal stopping time that is a single interval exit time. However, if $T$ is large or small enough, then one can find an optimal stopping time that is a simple exit time. In this case, at least one of the two points at which the process $Y$ is stopped is independent of the time constraint and the a priori probabilities.

The idea to interpret Bayesian sequential hypotheses testing as a stopping problem goes back to Wald (see [13]). Wald considers a problem version without expectation constraints and obtains optimal stopping rules that are exit times from intervals. This is in line with the well-known fact that in unconstrained one-dimensional optimal stopping problems with an infinite time horizon one can reduce the set of stopping times to first exit times of intervals (see e.g. Corollary 2.9 in [11]). In particular, the process at the stopping time has at most 2 mass points. Therefore, compared to the unconstrained sequential testing, the expectation constraint in 0.1 leads to a systematical difference in the optimal stopping rule.

Stopping at an interval exit time results in a test that is usually referred to as a sequential probability ratio test (SPRT). For testing two simple hypotheses on the drift rate of a Brownian motion a SPRT is optimal in the sense that the SPRT minimizes the expectation of the stopping time simultaneously under both hypotheses among all sequential tests having no larger errors of first and second kind, see e.g. Chapter 4 in [12]. In [8] the optimality of the SPRT has been extended for testing more general drift rates. Testing whether the filtered probability space $\left(\Omega, \mathcal{F},\left(\mathcal{F}_{t}\right), \mathbb{P}^{0}\right)$ or $\left(\Omega, \mathcal{F},\left(\mathcal{F}_{t}\right), \mathbb{P}^{1}\right)$ is realized is considered in [14] and [5. In this case, the SPRT with stopping rule $\tau$ is optimal in the sense that it minimizes the Kuhlback-Leibler divergence $\mathbb{E}^{i}\left[\log \left(\left.\frac{d \mathbb{P}^{i}}{d \mathbb{P}^{1-i}}\right|_{\mathcal{F}_{\tau}}\right)\right], i \in\{0,1\}$.

Recent results on Bayesian sequential analysis include [10, where the authors consider Bayesian sequential tests for two simple hypotheses on the mean reversion speed of an OrnsteinUhlenbeck process. Lisovskii [9] deals with sequentially testing two hypotheses on the drift rate of a Brownian bridge.

Some classical versions of the SPRT are described in the monographs [12] and [11. We postpone a more detailed comparison of the model in the present paper with the classical 
versions to the end of Section 2.2, after the rigorous description of our model and after stating the optimal stopping rule for the problem 0.1 .

There are only few articles considering stopping problems with expectation constraints. Kennedy [6] provides a solution method in a discrete time setting, based on Lagrangian techniques. Within a continuous time setting, the article [2] formulates a dynamic programming principle for stopping problems with expectation constraints and derives a verification theorem. Bayraktar and Yao [4] provide a proof of the dynamic programming principle and characterize the value function of the stopping problem as the unique viscosity solution of the associated fully non-linear Hamilton-Jacobi-Bellman equation.

\section{A Sequential Testing Model}

In this section we describe rigorously the sequential testing model with expectation constraints that we analyze in the article. We build upon the classical model in which two simple hypotheses on the drift of a Brownian motion are tested (see e.g. Chapter VI.21 in [11] or Chapter 4.2 in [12]).

Let $\left(\Omega, \mathcal{F},\left(\mathbb{P}^{y}\right)_{y \in[0,1]}\right)$ be a probability-statistical space. In the Bayesian formulation the probability measures $\mathbb{P}^{y}, y \in[0,1]$, are given by

$$
\mathbb{P}^{y}=y \mathbb{P}^{1}+(1-y) \mathbb{P}^{0} .
$$

Let $W=\left(W_{t}\right)_{t \in[0, \infty)}$ be a Brownian motion starting in 0 under every $\mathbb{P}^{y}$. Furthermore, let $\theta$ be a random variable independent of $W$ under every $\mathbb{P}^{y}$ with $\mathbb{P}^{y}[\theta=1]=y$ and $\mathbb{P}^{y}[\theta=0]=1-y$. Let

$$
X_{t}=\theta \kappa t+\sigma W_{t}, \quad t \in[0, \infty),
$$

where $\kappa \in \mathbb{R} \backslash\{0\}$ and $\sigma^{2}>0$. Then $\mathbb{P}^{y}[X \in \cdot \mid \theta=i]=\mathbb{P}^{i}[X \in \cdot], i \in\{0,1\}$, is the law of a Brownian motion with drift $i \kappa$ and diffusion coefficient $\sigma$. For $t \in[0, \infty)$ let $\mathcal{F}_{t}^{X}=\sigma\left(X_{s}: 0 \leq\right.$ $s \leq t)$ be the $\sigma$-algebra generated by $\left(X_{s}\right)_{s \in[0, t]}$. We suppose that an agent aims at deriving the value of $\theta$ by continuously observing the process $X$. Note that the a priori probabilities of the statistical hypotheses

$$
H_{0}: \theta=0 \text { and } H_{1}: \theta=1
$$

are given by $1-y$ and $y$ under $\mathbb{P}^{y}$. Define the posterior probability process $\left(Y_{t}\right)_{t \in[0, \infty)}$ by

$$
Y_{t}:=\mathbb{P}^{y}\left[\theta=1 \mid \mathcal{F}_{t}^{X}\right]
$$

According to Theorem 7.1 in [7] the likelihood ratio process $\left(\varphi_{t}\right)_{t \in[0, \infty)}$ defined as the RadonNikodým derivative of the measure $\mathbb{P}^{1}$ with respect to $\mathbb{P}^{0}$ on $\mathcal{F}_{t}^{X}$ satisfies

$$
\varphi_{t}:=\left.\frac{d \mathbb{P}^{1}}{d \mathbb{P}^{0}}\right|_{\mathcal{F}_{t}^{X}}=\exp \left(\frac{\kappa}{\sigma^{2}}\left(X_{t}-\frac{\kappa}{2} t\right)\right) .
$$

Moreover, we conclude from [12, p. 181] that

$$
Y_{t}=\left(\frac{y}{1-y} \varphi_{t}\right) /\left(1+\frac{y}{1-y} \varphi_{t}\right)
$$

and that $\left(Y_{t}\right)_{t \in[0, \infty)}$ solves

$$
d Y_{t}=\frac{\kappa}{\sigma} Y_{t}\left(1-Y_{t}\right) d \widetilde{W}_{t}, \quad Y_{0}=y,
$$


where

$$
\widetilde{W}_{t}=\frac{1}{\sigma}\left(X_{t}-\kappa \int_{0}^{t} Y_{s} d s\right)
$$

is a standard Brownian motion with respect to $\left(\mathcal{F}_{t}^{X}\right)$ and $\mathbb{P}^{y}$ (see Theorem 7.12 and Theorem 9.1 in [7]). Moreover, the filtration $\left(\mathcal{F}_{t}^{Y}\right)_{t \in[0, \infty)}$ generated by $\left(Y_{t}\right)_{t \in[0, \infty)}$ coincides with $\left(\mathcal{F}_{t}^{X}\right)_{t \in[0, \infty)}$. Observe that $\left(Y_{t}\right)_{t \in[0, \infty)}$ is a regular continuous strong Markov process under $\mathbb{P}^{y}$ with state space $J=(0,1)$. From (1.1) we conclude that if $Y_{t}$ is close to 0 or 1 , then the diffusion coefficient is small and hence the posterior probability is unlikely to change much in small time intervals.

We assume that the agent can accept $H_{0}$ only if the posterior probability $Y$ is smaller than or equal to a given threshold $\alpha \in\left(0, \frac{1}{2}\right)$. Similarly, the agent can accept $H_{1}$ only if $Y$ is greater than or equal to an upper threshold. For simplicity we set the upper threshold equal to $1-\alpha$.

Let $\beta \geq 1$ and $T \in[0, \infty)$. We define the payoff function by

$$
f(x)=\mathbb{1}_{(0, \alpha]}(x)+\beta \mathbb{1}_{[1-\alpha, 1)}(x), \quad x \in(0,1) .
$$

We assume that the agent aims at maximizing the expected payoff $\mathbb{E}^{y}\left[f\left(Y_{\tau}\right)\right]$ among all $\left(\mathcal{F}_{t}^{Y}\right)$ stopping times satisfying the expectation constraint $\mathbb{E}^{y}[\tau] \leq T$. The value function $V:[0, \infty) \times$ $(0,1) \rightarrow \mathbb{R}$ of the constrained optimal stopping problem is given by

$$
V(T, y)=\sup \left\{\mathbb{E}^{y}\left[f\left(Y_{\tau}\right)\right]: \tau\left(\mathcal{F}_{t}^{Y}\right) \text {-stopping time, } \mathbb{E}^{y}[\tau] \leq T\right\} .
$$

We now comment on the model assumptions. Notice that $f\left(Y_{\tau}\right)=0$ if $Y_{\tau} \in(\alpha, 1-\alpha)$. This reflects that the agent can neither accept $H_{0}$ nor $H_{1}$ if at the stopping time the posterior process is between the two thresholds $\alpha$ and $1-\alpha$. In other words, the agent gains nothing if she stops collecting data without any significant result.

Detecting a drift 0 or $\kappa$ can be of different value for the agent. Notice that the payoff function $f$ normalizes the gain for accepting $H_{0}$ to 1 and the gain from accepting $H_{1}$ to the possibly higher payoff $\beta \geq 1$.

The payoff function $f$ is of an all-or-nothing type. For a gain it only matters whether one of the thresholds is attained or not. We stress that the agent does not have to accept $H_{0}$ or $H_{1}$ as soon as $Y$ attains $\alpha$ or $1-\alpha$. She can continue observing the process $X$ and hope for significant results for the other hypothesis.

In the model version presented in Chapter VI.21, [11] and Chapter 4.2.1, [12] the agent chooses sequential decision rules $(\tau, d)$, where $\tau$ is an $\left(\mathcal{F}_{t}^{X}\right)$-stopping time and the decision rule $d$ is an $\mathcal{F}_{\tau}^{X}$-measurable random variable taking values in $\{0,1\}$. The decision rule $(\tau, d)$ tells the agent to stop at time $\tau$ and to accept hypothesis $H_{1}$ if $d=1$ and hypothesis $H_{0}$ if $d=0$. It is assumed that she wants to find a decision rule $\left(\tau^{*}, d^{*}\right)$ for which

$$
\mathbb{E}^{y}\left[\tau+a_{1} \mathbb{1}_{\{d=0, \theta=1\}}+a_{2} \mathbb{1}_{\{d=1, \theta=0\}}\right],
$$

$a_{1}, a_{2}>0$, is minimal. The minimization problem is then shown to be equivalent to the problem of finding a stopping time $\tau$ that minimizes $\mathbb{E}^{y}\left[\tau+a_{1} Y_{\tau} \wedge a_{2}\left(1-Y_{\tau}\right)\right]$. The constants $a_{1}$ and $a_{2}$ weigh $\mathbb{P}^{1}[d=0]$, the error of first kind, and $\mathbb{P}^{0}[d=1]$, the error of second kind.

In the model version of Chapter 4.2.1 in [12] the agent is assumed to minimize the expectation of the stopping time among all sequential decision rules for which the error of the first and second kind are bounded by given thresholds. Our model can be modified such that the thresholds on the posterior process can be endogenously determined from a given bound on the error of first and second kind. We explain this for a special case in Remark 2.3 below.

We close this section by introducing some notions and notation needed in the following chapters. 
First, we introduce an auxiliary optimal stopping problem, in which we only allow for interval exit times $\tau(a, b)=\inf \left\{t \in[0, \infty): Y_{t} \notin(a, b)\right\}, 0<a \leq b<1$, satisfying the expectation constraint. In this case, the law of $Y_{\tau}$ has at most two mass points. For $(T, y) \in[0, \infty) \times(0,1)$ let

$$
V_{2}(T, y)=\sup \left\{\mathbb{E}^{y}\left[f\left(Y_{\tau}\right)\right]: \mathbb{E}^{y}[\tau] \leq T, \operatorname{Law}\left(Y_{\tau}\right) \text { weighted sum of at most } 2 \text { Dirac measures }\right\} \text {. }
$$

It turns out that on a subset of the domain, but not the whole domain, $V_{2}$ coincides with $V$.

In Section 3 and Section 4 we show that if $V(T, y)>V_{2}(T, y)$, then there exists an optimal stopping time $\tau^{*}$ for 1.2 such that the law of $Y_{\tau^{*}}$ under $\mathbb{P}^{y}$ is a weighted sum of Dirac measures in $\alpha, b^{*}, 1-\alpha$, where $b^{*} \in\left(\alpha, \frac{1}{2}\right]$. The mass point $b^{*}$ is independent of $T$ and $y$. Hence, one can characterize an optimal stopping time for 1.2 with the same three points $\alpha, b^{*}, 1-\alpha$. The weights the optimal stopping time attributes to the three points, however, do depend on $T$ and $y$.

Secondly, for every $y \in(0,1)$ we introduce the function $q_{y}$ defined by

$$
q_{y}(x)= \begin{cases}\frac{2 \sigma^{2}}{\kappa^{2}}\left((2 x-1) \log \left(\frac{(1-y) x}{(1-x) y}\right)+\frac{1-2 y}{y(1-y)}(x-y)\right), & x \in(0,1), \\ \infty, & x \notin(0,1) .\end{cases}
$$

One can show that $q_{y}\left(Y_{t}\right)-t, t \in[0, \infty)$, is local martingale (see Theorem 2.1 in [3]). Moreover, the function $q_{y}$ allows to characterize the law of $Y_{\tau}$ for stopping times $\tau$ satisfying the expectation constraint $\mathbb{E}^{y}[\tau] \leq T$. In particular, we can reduce the optimal stopping problem (1.2) to a measure optimization problem which helps to prove our results, see Section 4.

If we impose that both hypotheses have probability $\frac{1}{2}$ at the beginning of the observation, i.e. $y=\frac{1}{2}$, then the function $q_{\frac{1}{2}}$ simplifies to

$$
q(x):=q_{\frac{1}{2}}(x)= \begin{cases}\frac{2 \sigma^{2}}{\kappa^{2}}(2 x-1) \log \left(\frac{x}{1-x}\right), & x \in(0,1), \\ \infty, & x \notin(0,1) .\end{cases}
$$

In particular, we have $q(x)=q(1-x), x \in \mathbb{R}$. For every $y \in(0,1)$ Equation (4) in [3] implies that

$$
q_{y}(x)=q(x)-q(y)-(x-y) q^{\prime}(y), \quad x \in(0,1) .
$$

In the following we first state the value function of the primal and the auxiliary optimal stopping problem for $y=\frac{1}{2}$ and examine the dependence of the value function and the optimal stopping times on the exogenous parameters $\alpha, \beta, \kappa$ and $\sigma$ in Section 2 . In Section 3 we collect the value functions $V_{2}$ and $V$ and the optimal stopping times for a general a priori probability of $\{\theta=1\}$ and prove these results in Section 4 .

\section{Optimal Stopping Rules when starting without Bias}

In this section we assume that the agent has no bias at the beginning of the observation process, i.e. $y=\frac{1}{2}$. First we state the value function and an optimal stopping time for the case where hypothesis 1 has a higher payoff. Then we focus on the case where both hypotheses yield the same payoff, i.e. $\beta=1$.

Since the a priori probability of $\{\theta=1\}$ is fixed, we write in the following $V(T)$ and $V_{2}(T)$ instead of $V\left(T, \frac{1}{2}\right)$ and $V_{2}\left(T, \frac{1}{2}\right)$, respectively. 


\section{1 $\quad H_{1}$ yields a higher Payoff}

Recall that the payoff of hypothesis $H_{1}$ is given by $\beta \geq 1$. Moreover, before the observation starts the agent believes that the drift 0 and $\kappa$ both occur with probability $\frac{1}{2}$.

Let $\tau(a, b)=\inf \left\{t \in[0, \infty): Y_{t} \notin(a, b)\right\}, a, b \in(0,1)$. Then one can show that for $0<a<$ $y<b<1$ it holds that $\mathbb{E}^{y}[\tau(a, b)]=\mathbb{E}^{y}\left[q_{y}\left(Y_{\tau(a, b)}\right)\right]$, see e.g. Lemma 2.2 in [3]. In particular, the expected time under $\mathbb{P}^{\frac{1}{2}}$ until the process $\left(Y_{t}\right)_{t \in[0, \infty)}$ hits either $\alpha$ or $1-\alpha$ for the first time is given by

$$
\mathbb{E}^{\frac{1}{2}}[\tau(\alpha, 1-\alpha)]=\mathbb{E}^{\frac{1}{2}}\left[q\left(Y_{\tau}\right)\right]=\frac{\frac{1}{2}-\alpha}{1-2 \alpha} q(\alpha)+\frac{\frac{1}{2}-\alpha}{1-2 \alpha} q(1-\alpha)=q(\alpha),
$$

If the upper bound for the expected time horizon is larger than $q(\alpha)$, then the agent obtains a payoff of $\frac{1}{2}(\beta+1)$ by stopping at $\alpha$ and $1-\alpha$. To increase the payoff, she can increase the probability to attain $1-\alpha$ by stopping at $1-\alpha$ and a point $a^{*} \in(0, \alpha]$ such that $\tau\left(a^{*}, 1-\alpha\right)$ exploits the full time horizon, i.e. $\mathbb{E}^{\frac{1}{2}}\left[\tau\left(a^{*}, 1-\alpha\right)\right]=T$.

If the time horizon is smaller than $q(\alpha)$, then two cases can occur. If the constraint is not too small, i.e. $T \in\left(T^{*}, q(\alpha)\right)$ for some $T^{*} \in[0, q(\alpha))$, then stopping at three points yields a higher payoff than stopping at two points. The optimal measure has mass points $\alpha, 1-\alpha$ and $b^{*} \in(\alpha, 1-\alpha)$, where $b^{*}$ is independent of the time constraint. If $T \leq T^{*}$, then stopping at two points is optimal. The optimal stopping rule is as follows: If the time constraint is too small to reach $b^{*}$ and $1-\alpha$ in expectation, then stop at $1-\alpha$ and a point $a^{*}=a^{*}(T) \in\left[b^{*}, \frac{1}{2}\right)$ such that $\mathbb{E}^{\frac{1}{2}}\left[\tau\left(a^{*}, 1-\alpha\right)\right]=T$. Moreover, the point $a^{*}$ decreases to $b^{*}$ for $T \nearrow T^{*}$.

For $T \in\left(T^{*}, q(\alpha)\right)$ it is optimal to use a consecutive exit time: First stop at $\alpha$ and $b_{1} \in$ $\left(\frac{1}{2}, 1-\alpha\right)$ and if the process attains $b_{1}$ before $\alpha$, then continue until the process either hits $b^{*}$ or $1-\alpha$.

In order to state the value function more precisely, let

- $b^{*} \in\left(\alpha, \frac{1}{2}\right]$ be the unique solution on $[\alpha, 1-\alpha]$ of

$$
\ell(b):=(\beta-1)(q(\alpha)-q(b))+(1-\alpha-b+b \beta-\alpha \beta) q^{\prime}(b)=0,
$$

- $T^{*}=\frac{\frac{1}{2}-b^{*}}{1-\alpha-b^{*}} q(\alpha)+\frac{\frac{1}{2}-\alpha}{1-\alpha-b^{*}} q\left(b^{*}\right)$,

- $a^{*}(T)$ be the unique solution of $\frac{\frac{1}{2}-\alpha}{1-\alpha-a} q(a)+\frac{\frac{1}{2}-a}{1-\alpha-a} q(\alpha)=T$ on $\left(0, \frac{1}{2}\right]$.

Now we can formulate the main result of this section.

Theorem 2.1. The value function $V$ of the optimal stopping problem (1.2) is given by

$$
V(T)= \begin{cases}V_{2}(T), & T \in\left[0, T^{*}\right] \cup[q(\alpha), \infty), \\ \frac{T-q\left(b^{*}\right)}{q(\alpha)-q\left(b^{*}\right)}+(\beta-1)\left(\frac{1}{2}-\frac{(q(\alpha)-T)\left(b^{*}-\alpha\right)}{(1-2 \alpha)\left(q(\alpha)-q\left(b^{*}\right)\right)}\right), & T \in\left(T^{*}, q(\alpha)\right),\end{cases}
$$

where

$$
V_{2}(T)= \begin{cases}\beta \frac{\frac{1}{2}-a^{*}(T)}{1-\alpha-a^{*}(T)}, & T \in[0, q(\alpha)), \\ 1+(\beta-1) \frac{\frac{1}{2}-a^{*}(T)}{1-\alpha-a^{*}(T)}, & T \geq q(\alpha)\end{cases}
$$


For $T \in\left[0, T^{*}\right] \cup[q(\alpha), \infty)$ the stopping time $\tau\left(a^{*}(T), 1-\alpha\right)$ is optimal for $V(T)$. For $T \in$ $\left(T^{*}, q(\alpha)\right)$ an optimal stopping time for $V(T)$ is given by

$$
\tau^{*}=\tau\left(\alpha, b_{1}\right)+\mathbb{1}_{\left\{Y_{\tau\left(\alpha, b_{1}\right)}=b_{1}\right\}} \inf \left\{t \in[0, \infty): Y_{\tau\left(\alpha, b_{1}\right)+t} \notin\left(b^{*}, 1-\alpha\right)\right\},
$$

where $b_{1} \in\left(\frac{1}{2}, 1-\alpha\right)$ is given by

$$
b_{1}=1-\alpha-\frac{\left.(1-2 \alpha)\left(1-\alpha-b^{*}\right)(q(\alpha)-T)\right)}{\left(\frac{3}{2}-2 \alpha-b^{*}\right) q(\alpha)-\left(\frac{1}{2}-\alpha\right) q\left(b^{*}\right)-\left(1-\alpha-b^{*}\right) T} .
$$

Here we do not provide a proof because the statement follows from more general results (Lemma 3.1 and Theorem 3.2 in Section 3.

If the payoff for hypothesis 1 is larger than for hypothesis 0 , then the expected payoff depends on three cases for the average time constraint. If the posterior probability process can attain, in expectation, $\alpha$ and $1-\alpha$ within the given time horizon, then it is optimal for the agent to wait until she either can accept $H_{1}$ for the first time or the posterior probability process falls below $a^{*}(T) \leq \alpha$. Although she can assume that the drift equals 0 if the posterior process is less or equal to $\alpha$, she continues observing the signals. This is due to the fact that she hopes for enough positive signals in the remaining time such that the observations suggest a drift $\kappa$. To maximize the payoff the agent always wants the signals to indicate a drift $\kappa$ and the posterior probability process to hit $1-\alpha$. In the case of very small time horizons, if a drift $\kappa$ seems unlikely given the first signals, then she quits observing the process with no result. Here unlikely means that the posterior probability for the drift being $\kappa$ is less or equal to $a^{*}(T)$.

Finally, if the time constraint is large enough but $Y$ cannot reach both $\alpha$ and $1-\alpha$ in expectation, then the agent stops observing the signals if the posterior probability process equals $\alpha$ or $1-\alpha$ for the first time or $b^{*}$ for the first time after it hits the level $b_{1} \in\left(\frac{1}{2}, 1-\alpha\right)$. In particular, this corresponds to stopping at a time $\tau^{*}$ if the agent is convinced that the drift is either 0 or $\kappa$ and she quits the observation process with no result if the posterior probability process first attains a level $b_{1}$ and then goes back to $b^{*}$ before hitting $1-\alpha$. Roughly speaking, she stops at $b^{*}$ if it takes too long to start the observation process afresh after it has first attained $b_{1}$, then falls below $\frac{1}{2}$ and attains $b^{*}$.

\subsection{Both Hypotheses have the same Payoff}

In this section we assume that both hypotheses yield the same payoff. If $\beta=1$, then the result of Section 2.1 simplifies.

Corollary 2.2. For $\beta=1$ the value function of the optimal stopping problem $(1.2)$ is given by

$$
V(T)= \begin{cases}\frac{T}{q(\alpha)}, & T<q(\alpha), \\ 1, & T \geq q(\alpha) .\end{cases}
$$

Let $T \in(0, q(\alpha))$. Then the following consecutive exit time $\tau^{*}$ is optimal in 1.2 .

$$
\tau^{*}=\tau\left(\alpha, b_{1}\right)+\mathbb{1}_{\left\{Y_{H\left(\alpha, b_{1}\right)}=b_{1}\right\}} \inf \left\{t \in[0, \infty): Y_{\tau\left(\alpha, b_{1}\right)+t} \notin\left(\frac{1}{2}, 1-\alpha\right)\right\},
$$

where $b_{1}=\frac{q(\alpha)-\alpha T}{2 q(\alpha)-T} \in\left(\frac{1}{2}, 1-\alpha\right)$.

Observe that for $\beta=1$ we only have two cases for the time constraint. If $T$ is not too big, i.e. if $T$ is smaller than the expected time to reach both $\alpha$ and $1-\alpha$, then the maximal expected payoff is not attained by a stopping time such that the process at the stopping time has two mass points. Three points are necessary: Similar to the case $\beta \geq 1$ the agent stops if the posterior probability process equals $\alpha$ or $1-\alpha$ for the first time or $\frac{1}{2}$ for the first time after it hits the level $b_{1}$. 
Remark 2.3. Fix $T \in(0, \infty)$. Now let $\alpha \in\left(0, \frac{1}{2}\right)$ such that $q(\alpha)>T$. For the optimal stopping time $\tau^{*}$ in $(1.2)$ the error $\Upsilon$ for accepting $H_{0}$ although $H_{1}$ is true (error of first kind) and the error $\Psi$ of accepting $H_{1}$ in case of a true hypothesis $H_{0}$ (error of second kind) are given by

$$
\Upsilon=\Upsilon(\alpha)=\mathbb{P}^{1}\left[Y_{\tau^{*}} \leq \alpha\right]=\frac{1-\left(\frac{q(\alpha)-(1-\alpha) T}{q(\alpha)-\alpha T}\right)^{\sigma^{2}}}{\left(\frac{1-\alpha}{\alpha}\right)^{\sigma^{2}}-\left(\frac{q(\alpha)-(1-\alpha) T}{q(\alpha)-\alpha T}\right)^{\sigma^{2}}}=\Psi=\Psi(\alpha)=\mathbb{P}^{0}\left[Y_{\tau^{*}} \geq 1-\alpha\right] .
$$

One can show that the errors $\Upsilon$ and $\Psi$ are strictly increasing in $\alpha$ and that for every $\delta \in\left(0, \frac{1}{2}\right)$ there exists $\alpha^{*}(\delta)$ such that the type I error $\Upsilon\left(\alpha^{*}(\delta)\right)$ and the type II error $\Psi\left(\alpha^{*}(\delta)\right)$ are equal to $\delta$. Thus, for the same prescribed type I and type II error we can determine a threshold $\alpha^{*}$ guaranteeing the given error for the optimal stopping time $\tau^{*}$.

\subsection{Dependence on the Parameters}

We examine how the optimal stopping rule and the expressions used to obtain the value function $V$ in Section 2.1 change in the parameters and interpret these changes. More precisely, we focus on the dependence of $q, a^{*}(T), V_{2}, b^{*}, T^{*}$ and $V$ on $\alpha, \beta, \kappa$ and $\sigma$. We first summarize and comment on the dependence on the parameters and prove them afterwards. We use $\uparrow$ and $\downarrow$ to show that the expression in the left column increases respectively decreases when the parameter increases. The symbol o represents that there is no dependence on the parameter.

\begin{tabular}{c||c|c|c|c} 
& $\alpha($ if $\beta>1)$ & $\alpha($ if $\beta=1)$ & $\beta$ & $\left|\frac{\kappa}{\sigma}\right|$ \\
\hline$q$ & $\circ$ & $\circ$ & $\circ$ & $\downarrow$ \\
$a^{*}(T)$ & $\downarrow$ & $\downarrow$ & $\circ$ & $\downarrow$ \\
$V_{2}(T)$ & $\uparrow$ & $\uparrow$ & $\uparrow$ & $\uparrow$ \\
$b^{*}$ & $\uparrow$ & $\circ$ & $\downarrow$ & $\circ$ \\
$T^{*}$ & $\downarrow$ & $\circ$ & $\uparrow$ & $\downarrow$ \\
$V(T)$ & $\uparrow$ & $\uparrow$ & $\uparrow$ & $\uparrow$
\end{tabular}

The threshold $\alpha$ allows the agent to assume that the drift is 0 if the posterior probability process is less than or equal to $\alpha$ and to presume that the drift equals $\kappa$ if the process is greater than or equal to $1-\alpha$. If $\alpha$ increases, then she can already decide earlier on the drift's value. Hence, the payoff function $f$ increases and, thus, the value functions $V_{2}$ and $V$ increase. Furthermore, if $\alpha$ increases, then stopping at three points yields a higher payoff than stopping at two points for smaller time horizon $T$, because of the increasing payoff function. This explains why $T^{*}$ decreases in $\alpha$.

If the gain for hypothesis 1 increases, i.e. $\beta$ increases, then the value functions $V_{2}$ and $V$ increase. The larger $\beta$, the more mass is assigned to the point $1-\alpha$. In particular, the agent accepts to quit collecting information without a result and hence, to obtain nothing in order to hit $1-\alpha$ with a higher probability and thus, to increase the expected payoff. Therefore, we have to impose a higher constraint on the average waiting time to allow for three possible outcomes, i.e. $T^{*}$ increases. Since the expected time until the posterior probability process attains $\alpha$ and $1-\alpha$ does not change, the length of the time interval for which three outcomes are optimal decreases in $\beta$.

The ratio $\frac{\kappa}{\sigma}$ is a measure for the strength of the observable signals: If $\kappa$ is large compared to the diffusion coefficient $\sigma$ of the process $X$, then the influence of a drift $\kappa$ will predominate 
and the agent can conclude the value of $\theta$ after a short observation time. On the other hand, if $\frac{\kappa}{\sigma}$ is small, it becomes more difficult for the agent to decide whether she observes a drift or the effect of the noise. Thus, if the strength of the signal increases, the value function increases. Observe that the function $q$ decreases in $\left|\frac{\kappa}{\sigma}\right|$. The third stopping point $b^{*}$ does not change in $\left|\frac{\kappa}{\sigma}\right|$, because all expressions are scaled with the same factor. Then the expected time to attain the three points $\alpha, b^{*}$ and $1-\alpha$ is smaller and thus, $T^{*}$ is decreasing. Since $q$ decreases in $\left|\frac{\kappa}{\sigma}\right|$, when using two consecutive exit times, the auxiliary stopping point can increase and the constraint is still satisfied. In particular, an increasing auxiliary stopping point $b_{1}$ implies that the mass in the optimal measure in $\alpha$ and $1-\alpha$ increases and hence, the payoff increases. Furthermore, since $b^{*}$ does not depend on the strength of the signal, the mass in $\alpha$ and $1-\alpha$ increase in $\left|\frac{\kappa}{\sigma}\right|$.

\subsubsection{Dependence on the Threshold $\alpha$}

The function $q$ is independent of $\alpha$, but note that $\mathbb{E}^{\frac{1}{2}}[\tau(\alpha, 1-\alpha)]=q(\alpha)$ decreases in $\alpha \in\left(0, \frac{1}{2}\right)$ with $\lim _{\alpha \downarrow 0} q(\alpha)=\infty$ and $q\left(\frac{1}{2}\right)=0$. The payoff function $f$ is increasing in $\alpha$, thus, also the value functions $V_{2}$ and $V$ increase in $\alpha$.

In the following we write $V_{2}^{\alpha}$ and $a^{*}(T, \alpha)$ to emphasize the dependence of $V_{2}$ and $a^{*}(T)$ on $\alpha$. First observe that

$$
\frac{\frac{1}{2}-\alpha}{1-\alpha-a} q(a)+\frac{\frac{1}{2}-a}{1-\alpha-a} q(\alpha)
$$

strictly decreases in $a \in\left(0, \frac{1}{2}\right)$ for fixed $\alpha \in\left(0, \frac{1}{2}\right)$ as well as in $\alpha$ for fixed $a$. Hence, for $0<\alpha<\gamma<\frac{1}{2}$ and $T \in(0, \infty)$ we have

$$
\begin{aligned}
T & =\frac{\frac{1}{2}-\alpha}{1-\alpha-a^{*}(T, \alpha)} q\left(a^{*}(T, \alpha)\right)+\frac{\frac{1}{2}-a^{*}(T, \alpha)}{1-\alpha-a^{*}(T, \alpha)} q(\alpha) \\
& >\frac{\frac{1}{2}-\gamma}{1-\gamma-a^{*}(T, \alpha)} q\left(a^{*}(T, \alpha)\right)+\frac{\frac{1}{2}-a^{*}(T, \alpha)}{1-\gamma-a^{*}(T, \alpha)} q(\gamma),
\end{aligned}
$$

which implies that $a^{*}(T, \gamma)<a^{*}(T, \alpha)$ by the definition of $a^{*}(T, \gamma)$. Thus, $a^{*}(T, \alpha)$ is strictly decreasing in $\alpha$. Furthermore, it holds that $a^{*}(T, \alpha) \rightarrow 0$ as $\alpha \nearrow \frac{1}{2}$. Indeed, let $\varepsilon \in\left(0, \frac{1}{2}\right)$ and assume that $a^{*}(T, \alpha) \geq \varepsilon$ for all $\alpha \in\left(0, \frac{1}{2}\right)$. Since (2.1) is decreasing in $a$, it follows that

$$
\begin{aligned}
T & =\frac{\frac{1}{2}-\alpha}{1-\alpha-a^{*}(T, \alpha)} q\left(a^{*}(T, \alpha)\right)+\frac{\frac{1}{2}-a^{*}(T, \alpha)}{1-\alpha-a^{*}(T, \alpha)} q(\alpha) \\
& \leq \frac{\frac{1}{2}-\alpha}{1-\alpha-\varepsilon} q(\varepsilon)+\frac{\frac{1}{2}-\varepsilon}{1-\alpha-\varepsilon} q(\alpha) \\
& \underset{\alpha \uparrow \frac{1}{2}}{\longrightarrow} 0 .
\end{aligned}
$$

Hence, $\lim _{\alpha \uparrow \frac{1}{2}} a^{*}(T, \alpha)=0$. Similarly, one shows that $\lim _{\alpha \downarrow 0} a^{*}(T, \alpha)=\frac{1}{2}$.

Since $\frac{\frac{1}{2}-a}{1-\alpha-a}$ strictly decreases in $a, a^{*}(T, \alpha)$ is strictly decreasing in $\alpha$ and $\frac{\frac{1}{2}-a}{1-\alpha-a}$ is strictly increasing in $\alpha$, we deduce for $0<\alpha<\gamma<\frac{1}{2}$ that

$$
\frac{\frac{1}{2}-a^{*}(T, \alpha)}{1-\alpha-a^{*}(T, \alpha)}<\frac{\frac{1}{2}-a^{*}(T, \gamma)}{1-\alpha-a^{*}(T, \gamma)}<\frac{\frac{1}{2}-a^{*}(T, \gamma)}{1-\gamma-a^{*}(T, \gamma)}
$$

Furthermore, it holds that $q(\gamma)<q(\alpha)$. Thus, for $T \in(0, q(\gamma))$ we have

$$
V_{2}^{\alpha}(T)=\beta \frac{\frac{1}{2}-a^{*}(T, \alpha)}{1-\alpha-a^{*}(T, \alpha)}<\beta \frac{\frac{1}{2}-a^{*}(T, \gamma)}{1-\gamma-a^{*}(T, \gamma)}=V_{2}^{\gamma}(T)
$$


Similarly, we conclude that $V_{2}^{\alpha}(T)<V_{2}^{\gamma}(T)$ for $T \in[q(\alpha), \infty)$. For $T \in[q(\gamma), q(\alpha))$ it holds that

$$
V_{2}^{\alpha}(T)=\beta \frac{\frac{1}{2}-a^{*}(T, \alpha)}{1-\alpha-a^{*}(T, \alpha)}<\beta \frac{\frac{1}{2}-a^{*}(T, \gamma)}{1-\gamma-a^{*}(T, \gamma)}+\frac{\frac{1}{2}-\gamma}{1-\gamma-a^{*}(T, \gamma)}=V_{2}^{\gamma}(T)
$$

To summarize, $V_{2}^{\alpha}(T)$ is strictly increasing in $\alpha$ for $T \in(0, \infty)$. In addition, we have that $\lim _{\alpha \downarrow 0} V_{2}^{\alpha}(T)=0$ and $\lim _{\alpha \uparrow \frac{1}{2}} V_{2}^{\alpha}(T)=\beta$.

To examine the dependence of $b^{*}$ on $\alpha$, we write $b^{*}(\alpha)$ and $\ell_{\alpha}$ instead of $b^{*}$ and $\ell$, respectively. Moreover, if $\beta>1$ we consider the modified equation

$$
\frac{\ell_{\alpha}(b)}{1-2 \alpha}=\frac{\beta-1}{1-2 \alpha}\left[q(\alpha)-q(b)+(b-\alpha) q^{\prime}(b)\right]+q^{\prime}(b)=0 .
$$

Then $b^{*}$ is a solution to $\ell_{\alpha}(b)=0$ if and only if $b^{*}$ is a solution to 2.2 . Notice that

$$
\frac{\partial}{\partial \alpha} \frac{\ell_{\alpha}(b)}{1-2 \alpha}=\frac{\beta-1}{(1-2 \alpha)^{2}}\left\{2\left[q(\alpha)-q(b)+(b-\alpha) q^{\prime}(\alpha)\right]-(1-2 b)\left[q^{\prime}(b)-q^{\prime}(\alpha)\right]\right\}<0
$$

for $b \in\left(\alpha, \frac{1}{2}\right]$ by the strict convexity of $q$. Now let $0<\alpha<\gamma<\frac{1}{2}$. Then 2.3) yields for all $b \in\left(\gamma, \frac{1}{2}\right)$ that

$$
\frac{\ell_{\alpha}(b)}{1-2 \alpha}>\frac{\ell_{\gamma}(b)}{1-2 \gamma}
$$

In particular, by 2.4 and since $\frac{\ell_{\gamma}(b)}{1-2 \gamma}$ is strictly increasing in $b$, we have for all $b \geq b^{*}(\gamma)$ that

$$
\frac{\ell_{\alpha}(b)}{1-2 \alpha}>\frac{\ell_{\gamma}\left(b^{*}(\gamma)\right)}{1-2 \gamma}=0
$$

Therefore, $b^{*}(\alpha)<b^{*}(\gamma)$, i.e. $b^{*}$ is strictly increasing in $\alpha$.

Since $\frac{\frac{1}{2}-a}{1-\alpha-a} q(\alpha)+\frac{\frac{1}{2}-\alpha}{1-\alpha-a} q(a)$ strictly decreases in $a$ for fixed $\alpha$ and in $\alpha$ for fixed $a$, it follow that $T^{*}$ decreases in $\alpha$. Indeed,

$$
\begin{aligned}
T^{*}(\alpha) & =\frac{\frac{1}{2}-b^{*}(\alpha)}{1-\alpha-b^{*}(\alpha)} q(\alpha)+\frac{\frac{1}{2}-\alpha}{1-\alpha-b^{*}(\alpha)} q\left(b^{*}(\alpha)\right) \\
& >\frac{\frac{1}{2}-b^{*}(\gamma)}{1-\alpha-b^{*}(\gamma)} q(\alpha)+\frac{\frac{1}{2}-\alpha}{1-\alpha-b^{*}(\gamma)} q\left(b^{*}(\gamma)\right) \\
& >\frac{\frac{1}{2}-b^{*}(\gamma)}{1-\gamma-b^{*}(\gamma)} q(\alpha)+\frac{\frac{1}{2}-\gamma}{1-\gamma-b^{*}(\gamma)} q\left(b^{*}(\gamma)\right)=T^{*}(\gamma)
\end{aligned}
$$

If $\beta=1$, then $b^{*}=\frac{1}{2}$ and $T^{*}=0$ are independent of $\alpha$.

\subsubsection{Dependence on $\beta$}

Observe that $q$ and $a^{*}(T)$ are independent of $\beta$. Since the payoff function $f$ is increasing in $\beta$, the functions $V_{2}$ and $V$ increase in $\beta$. Furthermore, $V_{2}(T)$ is linear in $\beta$, thus, it strictly increases in $\beta$ with $\lim _{\beta \rightarrow \infty} V_{2}(T)=\infty$ for $T \in(0, \infty)$ and for $\beta=1$ it holds that

$$
V_{2}(T)= \begin{cases}\frac{\frac{1}{2}-a^{*}(T)}{1-\alpha-a^{*}(T)}, & T \in[0, q(\alpha)), \\ 1, & T \geq q(\alpha) .\end{cases}
$$


For the dependence of $b^{*}$ on $\beta$ we use that

$$
\ell(b)=(\beta-1)(q(\alpha)-q(b))+(1-\alpha-b+b \beta-\alpha \beta) q^{\prime}(b)
$$

strictly increases in $b$ for fixed $\beta$ as well as in $\beta$ for fixed $b \in\left(\alpha, \frac{1}{2}\right]$. Therefore, $b^{*}$ strictly decreases in $\beta$. Furthermore, we have $b^{*}=\frac{1}{2}$ if $\beta=1$ and $b^{*} \downarrow \alpha$ for $\beta \rightarrow \infty$. To see the second claim, first note that $q^{\prime}$ strictly increases on $\left[\alpha, \frac{1}{2}\right]$ with $q^{\prime}\left(\frac{1}{2}\right)=0$. Thus, for $\beta>\frac{1}{2 \alpha}$ it holds that

$$
\begin{aligned}
\ell(b)=(\beta-1)\left[q(\alpha)-q(b)+(b-\alpha) q^{\prime}(b)\right] & +(1-2 \alpha) q^{\prime}(b) \\
& >(\beta-1)\left[q(\alpha)-q(b)+(b-\alpha) q^{\prime}(b)+\frac{q^{\prime}(\alpha)}{\beta}\right] .
\end{aligned}
$$

Using that $r(b):=q(\alpha)-q(b)+(b-\alpha) q^{\prime}(b)$ is strictly increasing on $\left[\alpha, \frac{1}{2}\right]$ and continuous with $r(\alpha)=0$ and $r\left(\frac{1}{2}\right)=q(\alpha)$, we conclude that for $\beta>\max \left\{\frac{-q^{\prime}(\alpha)}{q(\alpha)}, \frac{1}{2 \alpha}\right\}$ there exists $d(\beta):=r^{-1}\left(\frac{-q^{\prime}(\alpha)}{\beta}\right) \in\left(\alpha, \frac{1}{2}\right)$ such that

$$
r(d(\beta))+\frac{q^{\prime}(\alpha)}{\beta}=0
$$

In particular, for all $b>d(\beta)$ we have $r(b)>-\frac{q^{\prime}(\alpha)}{\beta}>0$, which together with 2.5 and the fact that $\ell$ is strictly increasing implies that $b^{*}=b^{*}(\beta) \in(\alpha, d(\beta))$. Since $\frac{q^{\prime}(\alpha)}{\beta} \rightarrow 0$ for $\beta \rightarrow \infty$, we conclude that $d(\beta) \downarrow \alpha$ and therefore, $b^{*}(\beta) \downarrow \alpha$ as $\beta \rightarrow \infty$.

For the dependence of $T^{*}$ on $\beta$ observe that $b \mapsto \frac{\frac{1}{2}-b}{1-\alpha-b} q(\alpha)+\frac{\frac{1}{2}-\alpha}{1-\alpha-b} q(b)$ is decreasing and $b^{*}$ decreases in $\beta$. Hence, it holds that $T^{*}$ increases in $\beta$ with $T^{*}=0$ if $\beta=1$ and $\lim _{\beta \rightarrow \infty} T^{*}=$ $q(\alpha)$.

\subsubsection{Dependence on the Strength of the Signal $\frac{\kappa}{\sigma}$}

First note that the expressions only depend on $\left|\frac{\kappa}{\sigma}\right|$. The function $q$ strictly decreases in $\left|\frac{\kappa}{\sigma}\right|$ on $(0,1) \backslash\left\{\frac{1}{2}\right\}$ and $q\left(\frac{1}{2}\right)=0$ for all $\left|\frac{\kappa}{\sigma}\right|>0$. To emphasize the dependence on $\left|\frac{\kappa}{\sigma}\right|$, we write $q^{\kappa / \sigma}$ instead of $q$ in the following. Using that $a^{*}(T), T \in[0, \infty)$, is the unique solution of

$$
T=\frac{\frac{1}{2}-\alpha}{1-\alpha-a} q^{\kappa / \sigma}(a)+\frac{\frac{1}{2}-a}{1-\alpha-a} q^{\kappa / \sigma}(\alpha)=\left|\frac{\sigma}{\kappa}\right|^{2}\left(\frac{\frac{1}{2}-\alpha}{1-\alpha-a} q^{1}(a)+\frac{\frac{1}{2}-a}{1-\alpha-a} q^{1}(\alpha)\right)
$$

we conclude that $a^{*}(T)$ solves

$$
\frac{\frac{1}{2}-\alpha}{1-\alpha-a} q^{1}(a)+\frac{\frac{1}{2}-a}{1-\alpha-a} q^{1}(\alpha)=\left|\frac{\kappa}{\sigma}\right|^{2} T .
$$

Since $\frac{\frac{1}{2}-\alpha}{1-\alpha-a} q^{1}(a)+\frac{\frac{1}{2}-a}{1-\alpha-a} q^{1}(\alpha)$ strictly decreases in $a \in\left(0, \frac{1}{2}\right)$, it holds that $a^{*}(T)$ strictly decreases in $\left|\frac{\kappa}{\sigma}\right|$ for $T \in(0, \infty)$. In addition, it holds that $a^{*}(T) \rightarrow \frac{1}{2}$ for $\left|\frac{\kappa}{\sigma}\right| \rightarrow 0$ and $a^{*}(T) \rightarrow 0$ for $\left|\frac{\kappa}{\sigma}\right| \rightarrow \infty$.

Using that $a \mapsto \frac{\frac{1}{2}-a}{1-\alpha-a}$ is strictly decreasing on $\left(0, \frac{1}{2}\right)$ we conclude that $V_{2}(T)$ is strictly increasing in $\left|\frac{\kappa}{\sigma}\right|$. The point $b^{*}$ is independent of the ratio $\frac{\kappa}{\sigma}$, because

$$
(\beta-1)\left[q^{\kappa / \sigma}(\alpha)-q^{\kappa / \sigma}(b)\right]+(1-\alpha-b+\beta b-\alpha \beta)\left(q^{\kappa / \sigma}\right)^{\prime}(b)=0
$$

holds if and only if

$$
(\beta-1)\left[q^{1}(\alpha)-q^{1}(b)\right]+(1-\alpha-b+\beta b-\alpha \beta)\left(q^{1}\right)^{\prime}(b)=0 .
$$


For $T^{*}$ we again use $q^{\kappa / \sigma}=\left|\frac{\sigma}{\kappa}\right|^{2} q^{1}$ to obtain

$$
T^{*}=\left|\frac{\sigma}{\kappa}\right|^{2}\left(\frac{\frac{1}{2}-\alpha}{1-\alpha-b^{*}} q^{1}\left(b^{*}\right)+\frac{\frac{1}{2}-b^{*}}{1-\alpha-b^{*}} q^{1}(\alpha)\right) .
$$

Therefore, $T^{*}$ is strictly decreasing in $\left|\frac{\kappa}{\sigma}\right|$ with $\lim _{|\kappa / \sigma| \rightarrow 0} T^{*}=\infty$ and $\lim _{|\kappa / \sigma| \rightarrow \infty} T^{*}=0$. Similarly, we deduce that $q^{\kappa / \sigma}(\alpha)-T^{*}$ decreases in $\left|\frac{\kappa}{\sigma}\right|$.

The auxiliary stopping point $b_{1}$ is given by

$$
\begin{aligned}
b_{1} & =1-\alpha-\frac{(1-2 \alpha)\left(1-\alpha-b^{*}\right)(q(\alpha)-T)}{\left(\frac{3}{2}-2 \alpha-b^{*}\right) q(\alpha)-\left(\frac{1}{2}-\alpha\right) q\left(b^{*}\right)-\left(1-\alpha-b^{*}\right) T} \\
& =1-\alpha-\frac{(1-2 \alpha)\left(1-\alpha-b^{*}\right)\left[q^{1}(\alpha)-q^{1}\left(a^{*}(T)\right)\right]}{\left(2-2 \alpha-b^{*}-a^{*}(T)\right) q^{1}(\alpha)-\left(1-\alpha-a^{*}(T)\right) q^{1}\left(b^{*}\right)-\left(1-\alpha-b^{*}\right) q^{1}\left(a^{*}(T)\right)},
\end{aligned}
$$

where we use that $a^{*}(T)$ solves $\frac{\frac{1}{2}-\alpha}{1-\alpha-a} q(a)+\frac{\frac{1}{2}-a}{1-\alpha-a} q(\alpha)=T$. Since $b^{*}$ is independent of $\left|\frac{\kappa}{\sigma}\right|$, we consider

$$
\begin{aligned}
& \frac{\partial}{\partial a}\left(-\frac{(1-2 \alpha)\left(1-\alpha-b^{*}\right)\left[q^{1}(\alpha)-q^{1}(a)\right]}{\left(2-2 \alpha-b^{*}-a\right) q^{1}(\alpha)-(1-\alpha-a) q^{1}\left(b^{*}\right)-\left(1-\alpha-b^{*}\right) q^{1}(a)}\right) \\
& \quad=-\frac{(1-2 \alpha)\left(1-\alpha-b^{*}\right)\left(q^{1}(\alpha)-q^{1}\left(b^{*}\right)\right)\left[q^{1}(\alpha)-q^{1}(a)-(1-\alpha-a)\left(q^{1}\right)^{\prime}(a)\right]}{\left[\left(2-2 \alpha-b^{*}-a\right) q^{1}(\alpha)-(1-\alpha-a) q^{1}\left(b^{*}\right)-\left(1-\alpha-b^{*}\right) q^{1}(a)\right]^{2}}<0 .
\end{aligned}
$$

Using that $a^{*}(T)$ decreases in $\left|\frac{\kappa}{\sigma}\right|$, the stopping point $b_{1}$ is increasing.

\section{Optimal Stopping Rules for general a priori Distributions}

In this section we state the main results for all a priori probabilities $y \in(0,1)$ of $\{\theta=1\}$. Hence, the posterior probability process $\left(Y_{t}\right)_{t \in[0, \infty)}$ starts in $y \in(0,1)$. The statements are proven in Section 4 .

Lemma 3.1. The value function of the optimal stopping problem (1.3) is given by

$$
V_{2}(T, y)= \begin{cases}\frac{c^{*}(T, y)-y}{c^{*}(T, y)-\alpha}, & y \in\left(\alpha, b^{*}\right], T \leq T^{*}(y), \\ \beta \frac{y-a^{*}(T, y)}{1-\alpha-a^{*}(T, y)}, & y \in\left(b^{*}, 1-\alpha\right), T \leq T^{*}(y), \\ \max \left\{\frac{c^{*}(T, y)-y}{c^{*}(T, y)-\alpha}, \beta \frac{y-a^{*}(T, y)}{1-\alpha-a^{*}(T, y)}\right\}, & y \in(\alpha, 1-\alpha), T \in\left(T^{*}(y), q(\alpha)-q(y)\right), \\ 1+(\beta-1) \frac{y-a^{*}(T, y)}{1-\alpha-a^{*}(T, y)}, & y \in(0, \alpha] \text { or } \\ \beta, & y \in(\alpha, 1-\alpha), T \geq q(\alpha)-q(y),\end{cases}
$$

where

- $b^{*} \in\left(\alpha, \frac{1}{2}\right]$ is the unique solution on $[\alpha, 1-\alpha]$ of

$$
\ell(b):=(\beta-1)(q(\alpha)-q(b))+(1-\alpha-b+b \beta-\alpha \beta) q^{\prime}(b)=0,
$$


- $a^{*}(T, y)$ is the unique solution of $\frac{1-\alpha-y}{1-\alpha-a} q(a)+\frac{y-a}{1-\alpha-a} q(\alpha)-q(y)=T$ on $(0, y]$,

- $c^{*}(T, y)$ is the unique solution of $\frac{c-y}{c-\alpha} q(\alpha)+\frac{y-\alpha}{c-\alpha} q(c)-q(y)=T$ on $[y, 1)$,

- $T^{*}(y)= \begin{cases}\frac{\left(b^{*}-y\right) q(\alpha)+(y-\alpha) q\left(b^{*}\right)}{b^{*}-\alpha}-q(y), & y \in\left(\alpha, b^{*}\right], \\ \frac{(1-\alpha-y) q\left(b^{*}\right)+\left(y-b^{*}\right) q(\alpha)}{1-\alpha-b^{*}}-q(y), & y \in\left(b^{*}, 1-\alpha\right] .\end{cases}$

Theorem 3.2. The value function $V$ of the optimal stopping problem 1.2 is given by

$$
V(T, y)=\left\{\begin{array}{cc}
\frac{T+q(y)-q\left(b^{*}\right)}{q(\alpha)-q\left(b^{*}\right)}+(\beta-1)\left(\frac{y-\alpha}{1-2 \alpha}-\frac{\left(b^{*}-\alpha\right)(q(\alpha)-T-q(y))}{(1-2 \alpha)\left(q(\alpha)-q\left(b^{*}\right)\right)}\right), \\
\quad y \in(\alpha, 1-\alpha), T \in\left(T^{*}(y), q(\alpha)-q(y)\right), \\
V_{2}(T, y), \quad \text { else. }
\end{array}\right.
$$

An optimal stopping time $\tau^{*}$ for $V(T, y)$ is given by

$$
\tau^{*}= \begin{cases}\tau\left(\alpha, c^{*}(T, y)\right), & y \in\left(\alpha, b^{*}\right], T \leq T^{*}(y), \\ \tau\left(a^{*}(T, y), 1-\alpha\right), & y \in(0, \alpha] \text { or } \\ & y \in\left(\alpha, b^{*}\right], T \geq q(\alpha)-q(y) \text { or } \\ & y \in\left(b^{*}, 1-\alpha\right), T \in\left[0, T^{*}(y)\right] \cup[q(\alpha)-q(y), \infty), \\ 0, & y \geq 1-\alpha .\end{cases}
$$

For $y \in(\alpha, 1-\alpha)$ and $T \in\left(T^{*}(y), q(\alpha)-q(y)\right)$ the stopping time

$$
\tau^{*}=\tau\left(\alpha, b_{1}\right)+\mathbb{1}_{\left\{Y_{\tau\left(\alpha, b_{1}\right)}=b_{1}\right\}} \inf \left\{t \in[0, \infty): Y_{\tau\left(\alpha, b_{1}\right)+t} \notin\left(b^{*}, 1-\alpha\right)\right\}
$$

is optimal in 1.2 , where

$$
b_{1}=1-\alpha-\frac{(1-2 \alpha)\left(1-\alpha-b^{*}\right)(q(\alpha)-T-q(y))}{\left(1-2 \alpha-b^{*}+y\right) q(\alpha)-(y-\alpha) q\left(b^{*}\right)-\left(1-\alpha-b^{*}\right)(T+q(y))} \in\left(b^{*} \vee y, 1-\alpha\right) .
$$

In particular, for $y \in(\alpha, 1-\alpha)$ and $T \in\left(T^{*}(y), q(\alpha)-q(y)\right)$ the law of $Y_{\tau^{*}}$ is purely atomic with mass points $\alpha, b^{*}$ and $1-\alpha$ that do not depend on $T$ nor $y$.

Remark 3.3. The value function $V_{2}(T, y)$ is not continuous in $(q(\alpha)-q(y), y)$ for $y \in(\alpha, 1-\alpha)$. Indeed, observe that $a^{*}(T, y)$ and $c^{*}(T, y)$ are continuous in $T$ for fixed $y$, because they are the inverse functions of a strictly decreasing respectively increasing and continuous function. Furthermore, we have $a^{*}(\bar{T}, y)=\alpha$ and $c^{*}(\bar{T}, y)=1-\alpha$, where $\bar{T}=q(\alpha)-q(y)$. Hence,

$$
\lim _{T \uparrow \bar{T}} V_{2}(T, y)=\max \left\{\frac{1-\alpha-y}{1-2 \alpha}, \beta \frac{y-\alpha}{1-2 \alpha}\right\}<\frac{1-\alpha-y}{1-2 \alpha}+\beta \frac{y-\alpha}{1-2 \alpha}=V_{2}(\bar{T}, y) .
$$

Remark 3.4. We can calculate $a^{*}(T, y), c^{*}(T, y)$ and $b^{*}$ numerically using Newton's method for given $\alpha \in\left(0, \frac{1}{2}\right), \beta \geq 1, y \in(0,1-\alpha)$ and $T \in[0, \infty)$.

In the following we state that for some average time constraints the expected payoff increases if we allow not only for two possible outcomes but for a third possibility, i.e. the law of the process at the stopping time has three mass points.

Corollary 3.5. Let $y \in(\alpha, 1-\alpha)$ and $T \in\left(T^{*}(y), q(\alpha)-q(y)\right)$, where $T^{*}(y)$ is defined in (3.2). Then

$$
V_{2}(T, y)<\sup \left\{\mathbb{E}^{y}\left[f\left(Y_{\tau}\right)\right]: \mathbb{E}^{y}[\tau] \leq T, Y_{\tau} \text { has at most } 3 \text { mass points }\right\}=V(T, y) .
$$




\section{Proof of Lemma 3.1, Theorem 3.2, and Corollary 3.5}

We show Lemma 3.1 and then use its statement to prove Theorem 3.2 . Corollary 3.5 follows from the proof of Theorem 3.2 .

First we reduce the optimal stopping problems $(1.2)$ and $(1.3)$ to measure optimization problems using the results obtained in [1]. Here $\mathcal{A}(T, y)$ denotes the set of all probability measures with support in $(0,1)$ such that $\int_{\mathbb{R}} x \mu(d x)=y$ and $\int_{\mathbb{R}} q_{y}(x) \mu(d x) \leq T$. Furthermore, let $\mathcal{A}_{n}(T, y)$ be the set of all discrete measures in $\mathcal{A}(T, y)$ with at most $n$ mass points, $n \in \mathbb{N}$. From Theorem 2.5 in [1] we conclude that

$$
\begin{gathered}
V(T, y)=\sup _{\mu \in \mathcal{A}_{3}(T, y)} \int_{\mathbb{R}} f(x) \mu(d x), \\
V_{2}(T, y)=\sup _{\mu \in \mathcal{A}_{2}(T, y)} \int_{\mathbb{R}} f(x) \mu(d x) .
\end{gathered}
$$

In the proof of Lemma 3.1 we only focus on $V_{2}$ as the value function of an optimal stopping problem. On the other hand, for the proof of Theorem 3.2 we use both the characterization of the value function as a measure optimization problem and as an optimal stopping problem. Changing the perspective allows to simplify and shorten the arguments.

Proof of Lemma 3.1. In the following we obtain the value of the optimal stopping problem (1.3). We consider the cases $y \in(0, \alpha], y \in(\alpha, 1-\alpha)$ and $y \in[1-\alpha, 1)$ separately. Furthermore, we assume that $T \in(0, \infty)$, because $V_{2}(0, y)=f(y)$ for all $y \in(0,1)$.

$y \in[1-\alpha, 1)$ : Since $f$ is bounded above by $\beta$, stopping immediately is optimal for $V_{2}(T, y)$, $T \in(0, \infty)$, and hence $V_{2}(T, y)=\beta$.

$y \in(0, \alpha]:$ Stopping directly yields a payoff of 1 . This is optimal for $\beta=1$. If $\beta>1$, then stopping at $1-\alpha$ and $a \in(0, y)$ has a higher payoff. Here $a$ has to be chosen in such a way that the stopping time satisfies the expectation constraint. Using the first exit time of $(b, c), b \in(0, y), c \in(1-\alpha, 1)$, is not optimal, because $f$ is constant on $(0, \alpha]$ and $[1-\alpha, 1)$, respectively. Indeed, once the process attains $1-\alpha$ the agent can obtain a payoff of $\beta$, but if she does not stop at $1-\alpha$, then with a positive probability the process goes below $1-\alpha$ and does not return to $1-\alpha$ within the given expected time.

Observe that in an optimal strategy the whole time horizon is exploited, because decreasing the point $a$, which entails a higher expected time for exiting $(a, 1-\alpha)$, increases the probability that the process hits $1-\alpha$ before $a$. Thus, it is sufficient to focus on $\tau(a, 1-\alpha)$ and choose $a$ such that $\mathbb{E}^{y}[\tau(a, 1-\alpha)]=T$. Lemma 2.2 in [3] implies that

$$
\mathbb{E}^{y}[\tau(a, 1-\alpha)]=\mathbb{E}^{y}\left[q_{y}\left(Y_{\tau(a, 1-\alpha)}\right)\right] .
$$

The law $\mu^{a}$ of $Y_{\tau(a, 1-\alpha)}$ is given by

$$
\mu^{a}=\frac{1-\alpha-y}{1-\alpha-a} \delta_{a}+\frac{y-a}{1-\alpha-a} \delta_{1-\alpha} .
$$

Now we determine $a$ such that

$$
\mathbb{E}^{y}[\tau(a, 1-\alpha)]=\int_{\mathbb{R}} q_{y}(x) \mu^{a}(d x)=\frac{1-\alpha-y}{1-\alpha-a} q_{y}(a)+\frac{y-a}{1-\alpha-a} q_{y}(1-\alpha)=T .
$$

Using (1.4) and $q(x)=q(1-x)$ we rewrite (4.3) as

$$
\int_{\mathbb{R}} q_{y}(x) \mu^{a}(d x)=\frac{1-\alpha-y}{1-\alpha-a} q(a)+\frac{y-a}{1-\alpha-a} q(\alpha)-q(y)=T .
$$


The map

$$
k_{y}:(0, y] \rightarrow[0, \infty), \quad a \mapsto \frac{1-\alpha-y}{1-\alpha-a} q(a)+\frac{y-a}{1-\alpha-a} q(\alpha)-q(y),
$$

is continuous and strictly decreasing, because

$$
k_{y}^{\prime}(a)=\frac{1-\alpha-y}{(1-\alpha-a)^{2}}\left(q(a)+(1-\alpha-a) q^{\prime}(a)-q(\alpha)\right)<0
$$

by the strict convexity of $q$ and since $q(\alpha)=q(1-\alpha)$. Moreover, it holds that $\lim _{a \downarrow 0} k_{y}(a)=\infty$ and $k_{y}(y)=0$. Hence, for every $T \in(0, \infty)$ there exists a unique $a^{*}(T, y)=k_{y}^{-1}(T) \in(0, y)$ such that 4.4 and, thus, 4.3 hold. Therefore,

$$
V_{2}(T, y)=\beta \frac{y-a^{*}(T, y)}{1-\alpha-a^{*}(T, y)} .
$$

$y \in(\alpha, 1-\alpha):$ Note that the expected time until the process $\left(Y_{t}\right)_{t \in[0, \infty)}$ hits either $\alpha$ or $1-\alpha$ for the first time is given by

$$
\mathbb{E}^{y}[\tau(\alpha, 1-\alpha)]=\mathbb{E}^{y}\left[q_{y}\left(Y_{\tau}\right)\right]=\frac{1-\alpha-y}{1-2 \alpha} q_{y}(\alpha)+\frac{y-\alpha}{1-2 \alpha} q_{y}(1-\alpha)=q(\alpha)-q(y) .
$$

The last equality follows from (1.4). Now we distinguish between the cases $T<q(\alpha)-q(y)$ and $T \geq q(\alpha)-q(y)$.

$T<q(\alpha)-q(y):$ In this case the process cannot reach both $\alpha$ and $1-\alpha$ within the given expected time horizon. Thus, at least one of the stopping points lies inside $(\alpha, 1-\alpha)$. Similar to the case where $y \in(0, \alpha]$, we conclude that either stopping at $\alpha$ and $c \in(y, 1-\alpha)$ such that $\mathbb{E}^{y}[\tau(\alpha, c)]=T$ or stopping at the first exit time of $(a, 1-\alpha), a \in(\alpha, y)$, with $\mathbb{E}^{y}[\tau(a, 1-\alpha)]=T$ is optimal. Here we cannot directly argue that the stopping rule $\tau(a, 1-\alpha)$ has a higher payoff than $\tau(\alpha, c)$, because if $T$ is small and $y$ is close to $\alpha$, then it may be better to stop at $\alpha$ than to wait until the process hits $1-\alpha$ with a small probability.

We now derive the stopping points $a$ and $c$. As in the case $y \in(0, \alpha]$ we conclude that there exists a unique $a^{*}(T, y)=k_{y}^{-1}(T) \in(0, y)$ such that $\mathbb{E}^{y}\left[\tau\left(a^{*}(T, y), 1-\alpha\right)\right]=T$. Furthermore, since $k_{y}$ is strictly decreasing and $T<q(\alpha)-q(y)=k_{y}(\alpha)$, it follows that $a^{*}(T, y) \in(\alpha, y)$. For the stopping point $c$ observe that the map

$$
g_{y}:[y, 1) \rightarrow[0, \infty), \quad c \mapsto \frac{c-y}{c-\alpha} q(\alpha)+\frac{y-\alpha}{c-\alpha} q(c)-q(y),
$$

is continuous and strictly increasing with $g_{y}(y)=0, \lim _{c \uparrow 1} g_{y}(c)=\infty$ and $g_{y}(1-\alpha)=$ $q(\alpha)-q(y)$. Therefore, there exists a unique $c^{*}(T, y)=g_{y}^{-1}(T) \in(y, 1-\alpha)$ such that $\mathbb{E}^{y}\left[\tau\left(\alpha, c^{*}(T, y)\right)\right]=T$. To sum up, we have

$$
V_{2}(T, y)=\max \left\{\frac{c^{*}(T, y)-y}{c^{*}(T, y)-\alpha}, \beta \frac{y-a^{*}(T, y)}{1-\alpha-a^{*}(T, y)}\right\} .
$$

Let $y \in\left(\alpha, b^{*}\right]$ and $T \leq T^{*}(y)=g_{y}\left(b^{*}\right)$, where $b^{*}$ is the unique solution of (3.1) on $[\alpha, 1-\alpha]$ (for existence and uniqueness see Lemma 5.2 in the appendix). In the following we write $a^{*}$ and $c^{*}$ instead of $a^{*}(T, y)$ and $c^{*}(T, y)$, respectively. We show that for $T \leq T^{*}(y)$ it holds that

$$
V_{2}(T, y)=\frac{c^{*}-y}{c^{*}-\alpha} \geq \beta \frac{y-a^{*}}{1-\alpha-a^{*}}
$$

with strict inequality if $\beta>1$. Assume that

$$
\beta \frac{y-a}{1-\alpha-a}>\frac{c^{*}-y}{c^{*}-\alpha}
$$


holds for some $a \in(\alpha, y)$. Then $a<y-\frac{(1-\alpha-y)\left(c^{*}-y\right)}{\beta\left(c^{*}-\alpha\right)-\left(c^{*}-y\right)}=: \bar{a}=\bar{a}(T, y)$, because $\frac{y-a}{1-\alpha-a}$ is strictly decreasing in $a$ and $\bar{a}$ is chosen in such a way that equality holds in 4.8 when replacing $a$ by $\bar{a}$. Observe that $\bar{a} \in(\alpha, y)$ if and only if $\beta>\frac{(1-2 \alpha)\left(c^{*}-y\right)}{(y-\alpha)\left(c^{*}-\alpha\right)}$. If $\bar{a} \leq \alpha$, then 4.8 does not hold for any $a \in(\alpha, y)$ and hence 4.7$)$ follows. If $\bar{a} \in(\alpha, y)$, then we show that $k_{y}(a)>g_{y}\left(c^{*}\right)=T$ for all $a \in(\alpha, \bar{a})$. In particular, we conclude that $a^{*} \geq \bar{a}$ and as a consequence (4.7) holds.

Since $T \leq T^{*}(y)=g_{y}\left(b^{*}\right)$ and $g_{y}$ is strictly increasing, it follows that $c^{*}=g_{y}^{-1}(T) \leq b^{*}$. First assume that $\beta>1$. Since $\ell$ is strictly increasing and we have $c^{*} \leq b^{*}$, it follows that $\ell\left(c^{*}\right)<\ell\left(b^{*}\right)=0$ and thus,

$$
q(\alpha)<q\left(c^{*}\right)-\frac{1-\alpha+c^{*}-c^{*} \beta-\alpha \beta}{\beta-1} q^{\prime}\left(c^{*}\right) .
$$

The definition of $\bar{a}, 4.9$ and the strict convexity of $q$ imply that

$$
\begin{aligned}
k_{y}(\bar{a})-g_{y}\left(c^{*}\right) & =-(\beta-1) \frac{y-\bar{a}}{1-\alpha-\bar{a}} q(\alpha)+\frac{1-\alpha-y}{1-\alpha-\bar{a}} q(\bar{a})-\left(1-\beta \frac{y-\bar{a}}{1-\alpha-\bar{a}}\right) q\left(c^{*}\right) \\
& >\frac{1-\alpha-y}{1-\alpha-\bar{a}}\left[q(\bar{a})-q\left(c^{*}\right)\right]+\frac{y-\bar{a}}{1-\alpha-\bar{a}}\left(1-\alpha-c^{*}+c^{*} \beta-\alpha \beta\right) q^{\prime}\left(c^{*}\right) \\
& >\left(\frac{1-\alpha-y}{1-\alpha-\bar{a}}\left(\bar{a}-c^{*}\right)+\frac{y-\bar{a}}{1-\alpha-\bar{a}}\left(1-\alpha-c^{*}+c^{*} \beta-\alpha \beta\right)\right) q^{\prime}\left(c^{*}\right) \\
& =0 .
\end{aligned}
$$

If $\beta=1$, then

$$
k_{y}(\bar{a})-g_{y}\left(c^{*}\right)=\frac{y-\alpha}{c^{*}-\alpha}\left[q(\bar{a})-q\left(c^{*}\right)\right] \geq \frac{y-\alpha}{c^{*}-\alpha}\left(\bar{a}-c^{*}\right) q^{\prime}\left(c^{*}\right) \geq 0,
$$

where we use that $\bar{a}<y<c^{*} \leq b^{*}=\frac{1}{2}$ and hence $q^{\prime}\left(c^{*}\right) \leq 0$. Since $k_{y}$ is strictly decreasing, it follows that $k_{y}(a)>k_{y}(\bar{a}) \geq g_{y}\left(c^{*}\right)=T$ for all $a \in(\alpha, \bar{a})$. Since $a^{*}=k_{y}^{-1}(T)$, we conclude that $a^{*} \geq \bar{a}$ and hence (4.7) holds. In particular, we have $V_{2}(T, y)=\frac{c^{*}-y}{c^{*}-\alpha}$ for $T \leq T^{*}(y)$. Similarly one can show that

$$
V_{2}(T, y)=\beta \frac{y-a^{*}(T, y)}{1-\alpha-a^{*}(T, y)}
$$

for $y \in\left(b^{*}, 1-\alpha\right)$ and $T \leq T^{*}(y)$.

$T \geq q(\alpha)-q(y)$ In this case the expected time to reach $\alpha$ and $1-\alpha$ is less or equal to $T$. For $T=q(\alpha)-q(y)$ the agent maximizes her payoff by stopping at $\alpha$ and $1-\alpha$. If $\beta>1$ and if $T$ increases, then she can increase the probability that the process attains $1-\alpha$ before hitting a lower bound $a \in\left[a^{*}(T, y), \alpha\right]$ by decreasing $a$. If she does so, the payoff increases. Since she gains more from increasing the probability at $1-\alpha$ than at $\alpha$, stopping at $a^{*}(T, y)$ and $1-\alpha$ is optimal. Therefore,

$$
V_{2}(T, y)=\frac{1-\alpha-y}{1-\alpha-a^{*}(T, y)}+\beta \frac{y-a^{*}(T, y)}{1-\alpha-a^{*}(T, y)}
$$

Proof of Theorem 3.2. First observe that $V(T, y) \geq V_{2}(T, y)$ for all $T \in[0, \infty)$ and $y \in(0,1)$. In this proof we also distinguish between the cases $y \in(0, \alpha], y \in(\alpha, 1-\alpha)$ and $y \in[1-\alpha, 1)$. Without loss of generality, we assume that $T \in(0, \infty)$.

$y \in[1-\alpha, 1):$ Since $f$ is bounded above by $\beta$, the stopping time $\tau^{*}=0$ is optimal. Hence 
$V(T, y)=V_{2}(T, y)$ for $T \in(0, \infty)$.

$y \in(0, \alpha]:$ If $\beta=1$, then we conclude as in the case $y \in[1-\alpha, 1)$ that stopping directly is optimal with $V(T, y)=V_{2}(T, y)=1$. If $\beta>1$, then we use the reformulations (4.1) and (4.2) as well as the fact that $V$ is the value function of the optimal stopping problem 1.2). To embed a measure $\mu$ with exactly three mass points $a<b<c$ the agent uses the following stopping rule $\tau$ (cf. the proof of Theorem 1.4 in [1]): She stops at the first exit time of $(a, d)$, where $d=(\mu(\{b\}) b+\mu(\{c\}) c) /(\mu(\{b\})+\mu(\{c\}))$ is an auxiliary stopping point. If $Y$ attains $a$ before $d$, then she is done; otherwise, she uses a second exit time, namely, she waits until the process equals $b$ or $c$.

From (4.1) we know that stopping at 3 points is enough. Let $\mu \in \mathcal{A}_{3}(T, y)$ with mass points $a<b<c \in(0,1)$, all having positive probability. Observe that it is not optimal if $a, b, c \in(0,1-\alpha)$, because then the payoff is less or equal to 1 , which is strictly smaller than $V_{2}(T, y)$. Moreover, two mass points in the interval $[1-\alpha, 1)$ cannot be optimal neither, because thinking in terms of the optimal stopping problem, the agent also gains $\beta$ by stopping at $1-\alpha$ but with a higher probability. Stopping at $a$ and $1-\alpha$ has a smaller expected value than stopping at $a, b$ and $c$ where $b, c \geq 1-\alpha$ and hence the stopping time $\tau(a, 1-\alpha)$ is admissible and yields a higher payoff than $\tau$. Similar to the proof of Lemma 3.1 we conclude that in an optimal measure it holds that $c=1-\alpha$. If $b \in(y, 1-\alpha)$, then, compared to the optimal strategy for $V_{2}(T, y)$, the probability to attain a payoff of $\beta$ is smaller. Indeed, for the payoff $\beta$ the process first has to hit the auxiliary stopping point $d$ and then attain $1-\alpha$. But if the agent additionally stops at $b$ after hitting $d$, the probability of hitting $1-\alpha$ decreases. Hence, $b \in(y, 1-\alpha)$ cannot be optimal. Finally, if $b \in(a, y]$, let

$$
\lambda=\frac{(d-y)(1-\alpha-b)}{(d-y)(1-\alpha-b)+(y-a)(1-\alpha-d)}
$$

and observe that $\lambda \in(0,1)$. Then stopping at the two points $r=\lambda a+(1-\lambda) b \in(a, y)$ and $1-\alpha$ has the same payoff than using the stopping time associated to $\mu$. Indeed, $\lambda$ is chosen such that $\mu(\{1-\alpha\})=\mathbb{P}^{y}\left[Y_{\tau(r, 1-\alpha)}=1-\alpha\right]$. Hence, the payoff of the two stopping rules coincides. Moreover, it holds that $(1-\mu(\{1-\alpha\})) \lambda=\mu(\{a\})$ and $(1-\mu(\{1-\alpha\}))(1-\lambda)=\mu(\{b\})$. The strict convexity of $q_{y}$ implies that

$$
\begin{aligned}
\mathbb{E}^{y}[\tau(r, 1-\alpha)] & =\mathbb{E}^{y}\left[q_{y}\left(Y_{\tau(r, 1-\alpha)}\right)\right] \\
& =\mathbb{P}^{y}\left[Y_{\tau(r, 1-\alpha)}=1-\alpha\right] q_{y}(\alpha)+\left(1-\mathbb{P}^{y}\left[Y_{\tau(r, 1-\alpha)}=1-\alpha\right]\right) q_{y}(r) \\
& <\mu(\{1-\alpha\}) q_{y}(\alpha)+(1-\mu(\{1-\alpha\}))\left(\lambda q_{y}(a)+(1-\lambda) q_{y}(b)\right) \\
& =\int_{\mathbb{R}} q_{y}(x) \mu(d x) \leq T .
\end{aligned}
$$

Therefore, the stopping time $\tau(r, 1-\alpha)$ is an admissible stopping time.

To sum up, this shows that using 3 points instead of 2 does not increase the value of the measure optimization problem (4.1) and thus, $V(T, y)=V_{2}(T, y)$.

$y \in(\alpha, 1-\alpha):$ Again we examine the cases $T<q(\alpha)-q(y)$ and $T \geq q(\alpha)-q(y)$ separately.

$T \geq q(\alpha)-q(y):$ Recall that $\mathbb{E}^{y}[\tau(\alpha, 1-\alpha)]=q(\alpha)-q(y)$. Therefore, both points $\alpha$ and $1-\alpha$ can be reached within the given time. Similar arguments as in the case $y \in(0, \alpha]$ show that $V(T, y)=V_{2}(T, y)$.

$T<q(\alpha)-q(y):$ Here the expected time constraint is to small to reach $\alpha$ and $1-\alpha$ within the given time horizon. Now we use the reformulation (4.1) and consider measures $\mu \epsilon$ $\mathcal{A}_{3}(T, y) \backslash \mathcal{A}_{2}(T, y)$. One can argue similarly to the case $y \in(0, \alpha]$ and conclude that it is 
sufficient to focus on measures $\mu$ with mass points $\alpha<b<1-\alpha$ and to use the full time horizon, i.e. $\int_{\mathbb{R}} q_{y}(x) \mu(d x)=T$. Note that the line of arguments interprets $V$ as the value of an optimal stopping problem. For every signed measure $\mu$ which is atomic and has three mass points, the constraints $\int_{\mathbb{R}} 1 \mu(d x)=1, \int_{\mathbb{R}} x \mu(d x)=y$ and $\int_{\mathbb{R}} q_{y}(x) \mu(d x)=T$ uniquely define the weights. To obtain a probability measure, one has to restrict the mass points to certain intervals. Let

$$
\begin{aligned}
\mu^{b}= & \frac{(1-\alpha-b)(T+q(y))+(b-y) q(\alpha)-(1-\alpha-y) q(b)}{(1-2 \alpha)(q(\alpha)-q(b))} \delta_{\alpha}+\frac{q(\alpha)-(T+q(y))}{q(\alpha)-q(b)} \delta_{b} \\
& +\frac{(b-\alpha)(T+q(y))-(b-y) q(\alpha)-(y-\alpha) q(b)}{(1-2 \alpha)(q(\alpha)-q(b))} \delta_{1-\alpha},
\end{aligned}
$$

where $b \in\left(k_{y}^{-1}(T), g_{y}^{-1}(T)\right)$ and the functions $k_{y}$ and $g_{y}$ are given by (4.5) and (4.6), respectively. By Lemma 5.1 in the appendix the measures $\mu^{b}, b \in\left(k_{y}^{-1}(T), g_{y}^{-1}(T)\right)$, are exactly all measures in $\mathcal{A}_{3}(\bar{T}, y) \backslash \mathcal{A}_{2}(T, y)$ such that the mass is concentrated in $\alpha, b$ and $1-\alpha$ and $\int_{\mathbb{R}} q_{y}(x) \mu^{b}(d x)=T$. We now maximize $\int_{\mathbb{R}} f(x) \mu^{b}(d x)$ over $b \in\left(k_{y}^{-1}(T), g_{y}^{-1}(T)\right) \subseteq(\alpha, 1-\alpha)$. Let

$$
n_{y}(b)=\int_{\mathbb{R}} f(x) \mu^{b}(d x)=\frac{T+q(y)-q(b)}{q(\alpha)-q(b)}+(\beta-1)\left(\frac{y-\alpha}{1-2 \alpha}-\frac{(b-\alpha)(q(\alpha)-T-q(y))}{(1-2 \alpha)(q(\alpha)-q(b))}\right) .
$$

Note that

$$
n_{y}^{\prime}(b)=-\frac{q(\alpha)-T-q(y)}{(1-2 \alpha)(q(\alpha)-q(b))^{2}}\left[(\beta-1)(q(\alpha)-q(b))+(1-\alpha-b+b \beta-\alpha \beta) q^{\prime}(b)\right] .
$$

Since $T<q(\alpha)-q(y)$ we conclude that for all $b \in\left(k_{y}^{-1}(T), g_{y}^{-1}(T)\right)$

$$
-\frac{q(\alpha)-T-q(y)}{(1-2 \alpha)(q(\alpha)-q(b))^{2}}<0 .
$$

Define the function $\ell:[\alpha, 1-\alpha] \rightarrow \mathbb{R}$ by

$$
\ell(b)=(\beta-1)(q(\alpha)-q(b))+(1-\alpha-b+b \beta-\alpha \beta) q^{\prime}(b) .
$$

Lemma 5.2 in the appendix implies that $\ell$ is strictly increasing and that there exists a unique $b^{*} \in\left(\alpha, \frac{1}{2}\right]$ such that $\ell\left(b^{*}\right)=0$. If $b^{*} \in\left(k_{y}^{-1}(T), g_{y}^{-1}(T)\right)$, then $b^{*}$ maximizes $n_{y}$ over $\left(k_{y}^{-1}(T), g_{y}^{-1}(T)\right)$. If $b^{*}<k_{y}^{-1}(T)$ or $b^{*}>g_{y}^{-1}(T)$, then $n_{y}$ is strictly decreasing respectively increasing on $\left(k_{y}^{-1}(T), g_{y}^{-1}(T)\right)$. To examine which conditions guarantee that $b^{*} \in$ $\left(k_{y}^{-1}(T), g_{y}^{-1}(T)\right)$ define

$$
T^{*}(y)= \begin{cases}g_{y}\left(b^{*}\right)=\frac{\left(b^{*}-y\right) q(\alpha)+(y-\alpha) q\left(b^{*}\right)}{b^{*}-\alpha}-q(y), & y \in\left(\alpha, b^{*}\right], \\ k_{y}\left(b^{*}\right)=\frac{(1-\alpha-y) q\left(b^{*}\right)+\left(y-b^{*}\right) q(\alpha)}{1-\alpha-b^{*}}-q(y), & y \in\left(b^{*}, 1-\alpha\right] .\end{cases}
$$

Observe that $T^{*}\left(b^{*}\right)=0$. Since $q\left(b^{*}\right)<q(\alpha)$ and $q$ is strictly convex, it follows that $T^{*}(y) \in$ $(0, q(\alpha)-q(y))$ for $y \neq b^{*}$. Let $y \in\left(\alpha, b^{*}\right]$. Using $k_{y}^{-1}(T)<y$, it holds that $b^{*}>k_{y}^{-1}(T)$. Moreover, since $g_{y}$ is strictly increasing it follows that $b^{*}<g_{y}^{-1}(T)$ if and only if $T^{*}(y)=$ $g_{y}\left(b^{*}\right)<T$. 
$T \leq T^{*}(y)$ : Then it holds that $b^{*}>g_{y}^{-1}(T)$. Thus, $n_{y}$ is strictly increasing and

$$
\begin{aligned}
\sup & \left\{n_{y}(b): b \in\left(k_{y}^{-1}(T), g_{y}^{-1}(T)\right)\right\} \\
& =n_{y}\left(g_{y}^{-1}(T)\right) \\
& =\frac{T+q(y)-q\left(g_{y}^{-1}(T)\right)}{q(\alpha)-q\left(g_{y}^{-1}(T)\right)}+(\beta-1)\left(\frac{y-\alpha}{1-2 \alpha}-\frac{\left(g_{y}^{-1}(T)-\alpha\right)(q(\alpha)-T-q(y))}{(1-2 \alpha)\left(q(\alpha)-q\left(g_{y}^{-1}(T)\right)\right.}\right) .
\end{aligned}
$$

Recall that $g_{y}^{-1}(T)=c^{*}(T, y)$ is the unique solution to $g_{y}(c)=T$ on $[y, 1)$. Hence, we conclude that

$$
\begin{aligned}
q(\alpha)-T-q(y) & =\frac{y-\alpha}{c^{*}(T, y)-\alpha}\left(q(\alpha)-q\left(c^{*}(T, y)\right)\right), \\
\frac{T+q(y)-q\left(c^{*}(T, y)\right)}{q(\alpha)-q\left(c^{*}(T, y)\right)} & =\frac{c^{*}(T, y)-y}{c^{*}(T, y)-\alpha} .
\end{aligned}
$$

By (4.11) the second summand in 4.10 equals 0. In addition, using 4.12 we conclude that $n_{y}\left(g_{y}^{-1}(T)\right)$ simplifies to

$$
n_{y}\left(g_{y}^{-1}(T)\right)=\frac{T+q(y)-q\left(g_{y}^{-1}(T)\right)}{q(\alpha)-q\left(g_{y}^{-1}(T)\right)}=\frac{T+q(y)-q\left(c^{*}(T, y)\right)}{q(\alpha)-q\left(c^{*}(T, y)\right)}=\frac{c^{*}(T, y)-y}{c^{*}(T, y)-\alpha} .
$$

Therefore, if $y \in\left(\alpha, b^{*}\right]$ and $T \leq T^{*}(y)$, then

$$
\sup \left\{\int_{\mathbb{R}} f(x) \mu^{b}(d x): b \in\left(k_{y}^{-1}(T), g_{y}^{-1}(T)\right)\right\}=\frac{c^{*}(T, y)-y}{c^{*}(T, y)-\alpha}=V_{2}(T, y) .
$$

Thus, $V(T, y)=V_{2}(T, y)$ and we do not gain more from stopping at 3 points than at 2 points. $T \in\left(T^{*}(y), q(\alpha)-q(y)\right):$ In this case it holds that $b^{*} \in\left(k_{y}^{-1}(T), g_{y}^{-1}(T)\right)$ and hence,

$$
\begin{aligned}
\sup \left\{\int_{\mathbb{R}} f(x) \mu^{b}(d x)\right. & \left.: b \in\left(k_{y}^{-1}(T), g_{y}^{-1}(T)\right)\right\}=n_{y}\left(b^{*}\right) \\
& =\frac{T+q(y)-q\left(b^{*}\right)}{q(\alpha)-q\left(b^{*}\right)}+(\beta-1)\left(\frac{y-\alpha}{1-2 \alpha}-\frac{\left(b^{*}-\alpha\right)(q(\alpha)-T-q(y))}{(1-2 \alpha)\left(q(\alpha)-q\left(b^{*}\right)\right)}\right) .
\end{aligned}
$$

It remains to show that $V(T, y)=n_{y}\left(b^{*}\right)>V_{2}(T, y)$ for $T \in\left(T^{*}(y), q(\alpha)-q(y)\right)$. In the following we write $a^{*}=k_{y}^{-1}(T)$ and $c^{*}=g_{y}^{-1}(T)$ instead of $a^{*}(T, y)$ and $c^{*}(T, y)$, respectively. 
4.11) and 4.12 imply that

$$
\begin{aligned}
n_{y}\left(b^{*}\right) & -\frac{c^{*}-y}{c^{*}-\alpha} \\
& =\frac{T+q(y)-q\left(b^{*}\right)}{q(\alpha)-q\left(b^{*}\right)}+(\beta-1)\left(\frac{y-\alpha}{1-2 \alpha}-\frac{\left(b^{*}-\alpha\right)(q(\alpha)-T-q(y))}{(1-2 \alpha)\left(q(\alpha)-q\left(b^{*}\right)\right)}\right)-\frac{c^{*}-y}{c^{*}-\alpha} \\
& =\frac{y-\alpha}{c^{*}-\alpha}\left(\frac{q\left(c^{*}\right)-q\left(b^{*}\right)}{q(\alpha)-q\left(b^{*}\right)}+(\beta-1) \frac{\left(c^{*}-b^{*}\right)\left[q(\alpha)-q\left(b^{*}\right)\right]+\left(b^{*}-\alpha\right)\left[q\left(c^{*}\right)-q\left(b^{*}\right)\right]}{(1-2 \alpha)\left(q(\alpha)-q\left(b^{*}\right)\right)}\right) \\
& =\frac{(y-\alpha)\left\{\left(c^{*}-b^{*}\right)(\beta-1)\left(q(\alpha)-q\left(b^{*}\right)\right)+\left(1-2 \alpha+(\beta-1)\left(b^{*}-\alpha\right)\right)\left[q\left(c^{*}\right)-q\left(b^{*}\right)\right]\right\}}{(1-2 \alpha)\left(c^{*}-\alpha\right)\left(q(\alpha)-q\left(b^{*}\right)\right)} \\
& =\frac{(y-\alpha)\left(1-\alpha-b^{*}+b^{*} \beta-\alpha \beta\right)\left[q\left(c^{*}\right)-q\left(b^{*}\right)-\left(c^{*}-b^{*}\right) q^{\prime}\left(b^{*}\right)\right]}{(1-2 \alpha)\left(c^{*}-\alpha\right)\left(q(\alpha)-q\left(b^{*}\right)\right)} \\
& >0,
\end{aligned}
$$

where the last equality follows from $\ell\left(b^{*}\right)=0$, i.e.

$$
(\beta-1)\left(q(\alpha)-q\left(b^{*}\right)\right)=-\left(1-\alpha-b^{*}+b^{*} \beta-\alpha \beta\right) q^{\prime}\left(b^{*}\right),
$$

and the strict convexity of $q$ implies the inequality.

Similar arguments lead to

$$
\begin{aligned}
n_{y}\left(b^{*}\right) & -\beta \frac{y-a^{*}}{1-\alpha-a^{*}} \\
& =\frac{\left(1-\alpha-a^{*}\right)\left(1-\alpha-b^{*}+b^{*} \beta-\alpha \beta\right)\left[q\left(a^{*}\right)-q\left(b^{*}\right)+\left(b^{*}-a^{*}\right) q^{\prime}\left(b^{*}\right)\right]}{(1-2 \alpha)\left(1-\alpha-a^{*}\right)\left(q(\alpha)-q\left(b^{*}\right)\right)}>0 .
\end{aligned}
$$

Hence,

$$
V(T, y)=n_{y}\left(b^{*}\right)>\max \left\{\frac{c^{*}-y}{c^{*}-\alpha}, \beta \frac{y-a^{*}}{1-\alpha-a^{*}}\right\}=V_{2}(T, y)
$$

for $y \in\left(\alpha, b^{*}\right]$ and $T \in\left(T^{*}(y), q(\alpha)-q(y)\right)$. For $y \in\left(b^{*}, 1-\alpha\right)$ we argue similarly to obtain

$$
V(T, y)= \begin{cases}V_{2}(T, y), & T \leq T^{*}(y), \\ \frac{T+q(y)-q\left(b^{*}\right)}{q(\alpha)-q\left(b^{*}\right)}+(\beta-1)\left(\frac{y-\alpha}{1-2 \alpha}-\frac{\left(b^{*}-\alpha\right)(q(\alpha)-T-q(y))}{(1-2 \alpha)\left(q(\alpha)-q\left(b^{*}\right)\right)}\right), & T \in\left(T^{*}(y), q(\alpha)-q(y)\right) .\end{cases}
$$

In particular, it holds that $V_{2}(T, y)<V(T, y)$ for $y \in\left(b^{*}, 1-\alpha\right)$ and $T \in\left(T^{*}(y), q(\alpha)-q(y)\right)$.

The optimal measure for the reformulated problem (4.1) for $y \in(\alpha, 1-\alpha)$ and $T \in$ $\left(T^{*}(y), q(\alpha)-q(y)\right)$ is given by

$$
\begin{aligned}
\mu^{b^{*}}= & \frac{\left(1-\alpha-b^{*}\right)(T+q(y))+\left(b^{*}-y\right) q(\alpha)-(1-\alpha-y) q\left(b^{*}\right)}{(1-2 \alpha)\left(q(\alpha)-q\left(b^{*}\right)\right)} \delta_{\alpha}+\frac{q(\alpha)-T-q(y)}{q(\alpha)-q\left(b^{*}\right)} \delta_{b^{*}} \\
& +\frac{\left(b^{*}-\alpha\right)(T+q(y))-\left(b^{*}-y\right) q(\alpha)-(y-\alpha) q\left(b^{*}\right)}{(1-2 \alpha)\left(q(\alpha)-q\left(b^{*}\right)\right)} \delta_{1-\alpha} .
\end{aligned}
$$


According to Theorem 1.4 in [1] and its proof, a stopping time $\tau^{*}$, which embeds $\mu^{b^{*}}$ in $\left(Y_{t}\right)_{t \in[0, \infty)}$ for $y \in(\alpha, 1-\alpha)$ and $T \in\left(T^{*}(y), q(\alpha)-q(y)\right)$ is given by

$$
\tau^{*}=\tau\left(\alpha, b_{1}\right)+\mathbb{1}_{\left\{Y_{\tau\left(\alpha, b_{1}\right)}=b_{1}\right\}} \inf \left\{t \in[0, \infty): Y_{\tau\left(\alpha, b_{1}\right)+t} \notin\left(b^{*}, 1-\alpha\right)\right\}
$$

for $b_{1} \in\left(b^{*} \vee y, 1-\alpha\right)$ such that $\mu^{b^{*}}(\{\alpha\})=\frac{b_{1}-y}{b_{1}-\alpha}$. Hence, we conclude that

$$
b_{1}=1-\alpha-\frac{(1-2 \alpha)\left(1-\alpha-b^{*}\right)(q(\alpha)-T-q(y))}{\left(1-2 \alpha-b^{*}+y\right) q(\alpha)-(y-\alpha) q\left(b^{*}\right)-\left(1-\alpha-b^{*}\right)(T+q(y))} .
$$

Similar arguments apply for $y \in\left(b^{*}, 1-\alpha\right)$ and $T \in\left(T^{*}(y), q(\alpha)-q(y)\right)$.

Remark 4.1. The optimal measure $\mu^{b^{*}}$ for $V(T, y)$ with $y \in(\alpha, 1-\alpha)$ and $T \in\left(T^{*}(y), q(\alpha)-q(y)\right)$ concentrates its mass in the points $\alpha, 1-\alpha$ and $b^{*}$. In particular, only the probability weights depend on $T$ and $y$ but not the mass points. Hence, the same three points suffice in 4.1.

Proof of Corollary 3.5. Follows directly from the proof of Theorem 3.2 .

Remark 4.2. In the proof of Lemma 3.1 we show that

$\max \left\{\frac{c^{*}(T, y)-y}{c^{*}(T, y)-\alpha}, \beta \frac{y-a^{*}(T, y)}{1-\alpha-a^{*}(T, y)}\right\}= \begin{cases}\frac{c^{*}(T, y)-y}{c^{*}(T, y)-\alpha}, & y \in\left(\alpha, b^{*}\right], T \leq T^{*}(y), \\ \beta \frac{y-a^{*}(T, y)}{1-\alpha-a^{*}(T, y)}, & y \in\left[b^{*}, 1-\alpha\right), T \leq T^{*}(y),\end{cases}$

where $b^{*}$ is the unique solution of (3.1) and $T^{*}(y)$ is given by $(3.2)$.

For $T \in\left(T^{*}(y), q(\alpha)-q(y)\right)$ it is in general not so easy to decide which value the maximum attains.

We now show how to differentiate $a^{*}(T, y)$ and $c^{*}(T, y)$ with respect to $y$. Let $\varphi \in \mathcal{C}^{1,1}(A \times$ $Y,(0, \infty))$ for $A, Y \subseteq \mathbb{R}$ open and assume that for fixed $y \in Y$ the mapping $\varphi_{y}: A \rightarrow[0, \infty), a \mapsto$ $\varphi(a, y)$, is bijective. Then for $T \in(0, \infty)$ it holds that

$$
\frac{\partial}{\partial T} \varphi_{y}^{-1}(T)=\frac{1}{\varphi_{y}^{\prime}\left(\varphi_{y}^{-1}(T)\right)}
$$

For the partial derivative with respect to $y$ observe that

$$
\begin{aligned}
0 & =\frac{\mathbf{d}}{\mathbf{d} y} \varphi_{y}\left(\varphi_{y}^{-1}(T)\right)=\frac{\mathbf{d}}{\mathbf{d} y} \varphi\left(\varphi_{y}^{-1}(T), y\right) \\
& =\left(\frac{\partial}{\partial a} \varphi\right)\left(\varphi_{y}^{-1}(T), y\right)\left(\frac{\partial}{\partial y} \varphi_{y}^{-1}(T)\right)+\left(\frac{\partial}{\partial y} \varphi\right)\left(\varphi_{y}^{-1}(T), y\right)
\end{aligned}
$$

Hence, it follows that

$$
\frac{\partial}{\partial y} \varphi_{y}^{-1}(T)=-\frac{\left(\frac{\partial}{\partial y} \varphi\right)\left(\varphi_{y}^{-1}(T), y\right)}{\varphi_{y}^{\prime}\left(\varphi_{y}^{-1}(T)\right)}
$$

As a consequence,

$$
\begin{aligned}
\frac{\partial}{\partial y} a^{*}(T, y) & =\frac{\left(1-\alpha-a^{*}(T, y)\right)\left[q(\alpha)-q\left(a^{*}(T, y)\right)-\left(1-\alpha-a^{*}(T, y)\right) q^{\prime}(y)\right]}{(1-\alpha-y)\left[q(\alpha)-q\left(a^{*}(T, y)\right)-\left(1-\alpha-a^{*}(T, y)\right) q^{\prime}\left(a^{*}(T, y)\right)\right]} \\
\frac{\partial}{\partial y} c^{*}(T, y) & =\frac{\left(c^{*}(T, y)-\alpha\right)\left[q(\alpha)-q\left(c^{*}(T, y)\right)+\left(c^{*}(T, y)-\alpha\right) q^{\prime}(y)\right]}{(y-\alpha)\left[q(\alpha)-q\left(c^{*}(T, y)\right)+\left(c^{*}(T, y)-\alpha\right) q^{\prime}\left(c^{*}(T, y)\right)\right]}
\end{aligned}
$$


Using the derivatives of $a^{*}(T, y)$ and $c^{*}(T, y)$ with respect to $y$ results in

$$
\begin{aligned}
& \frac{\partial}{\partial y}\left(\beta \frac{y-a^{*}(T, y)}{1-\alpha-a^{*}(T, y)}-\frac{c^{*}(T, y)-y}{c^{*}(T, y)-\alpha}\right) \\
& \quad=\frac{\beta\left[q^{\prime}(y)-q^{\prime}\left(a^{*}\right)\right]}{q(\alpha)-q\left(a^{*}\right)-\left(1-\alpha-a^{*}\right) q^{\prime}\left(a^{*}\right)}+\frac{q^{\prime}\left(c^{*}\right)-q^{\prime}(y)}{q(\alpha)-q\left(c^{*}\right)+\left(c^{*}-\alpha\right) q^{\prime}\left(c^{*}\right)}>0,
\end{aligned}
$$

where $a^{*}=a^{*}(T, y)$ and $c^{*}=c^{*}(T, y)$. Here we use that $q$ is strictly convex and $a^{*}(T, y)<y<$ $c^{*}(T, y)$. Moreover, for $y=\frac{1}{2}$ it holds that $c^{*}\left(T, \frac{1}{2}\right)=1-a^{*}\left(T, \frac{1}{2}\right)$ and thus

$$
\beta \frac{\frac{1}{2}-a^{*}\left(T, \frac{1}{2}\right)}{1-\alpha-a^{*}\left(T, \frac{1}{2}\right)}-\frac{c^{*}\left(T, \frac{1}{2}\right)-\frac{1}{2}}{c^{*}\left(T, \frac{1}{2}\right)-\alpha}=(\beta-1) \frac{\frac{1}{2}-a^{*}\left(T, \frac{1}{2}\right)}{1-\alpha-a^{*}\left(T, \frac{1}{2}\right)} \geq 0 .
$$

Therefore, for $y \geq \frac{1}{2}$ we have for all $T \in[0, \infty)$

$$
\max \left\{\frac{c^{*}(T, y)-y}{c^{*}(T, y)-\alpha}, \beta \frac{y-a^{*}(T, y)}{1-\alpha-a^{*}(T, y)}\right\}=\beta \frac{y-a^{*}(T, y)}{1-\alpha-a^{*}(T, y)} .
$$

\section{Appendix}

We prove two auxiliary results for the proof of Lemma 3.1 and Theorem 3.2. We first characterize all probability measures $\mu \in \mathcal{A}_{3}(T, y) \backslash \mathcal{A}_{2}(T, y)$ with mass points $\alpha, 1-\alpha$ and $b \in(\alpha, 1-\alpha)$ such that $\int_{\mathbb{R}} q_{y}(x) \mu(d x)=T$.

Lemma 5.1. Let $y \in(\alpha, 1-\alpha), T \in(0, q(\alpha)-q(y))$ and

$$
\begin{aligned}
\mu^{b}= & \frac{(1-\alpha-b)(T+q(y))+(b-y) q(\alpha)-(1-\alpha-y) q(b)}{(1-2 \alpha)(q(\alpha)-q(b))} \delta_{\alpha}+\frac{q(\alpha)-T-q(y)}{q(\alpha)-q(b)} \delta_{b} \\
& +\frac{(b-\alpha)(T+q(y))-(b-y) q(\alpha)-(y-\alpha) q(b)}{(1-2 \alpha)(q(\alpha)-q(b))} \delta_{1-\alpha},
\end{aligned}
$$

where $b \in\left(k_{y}^{-1}(T), g_{y}^{-1}(T)\right)$ and the functions $k_{y}$ and $g_{y}$ are given by (4.5) and 4.6), respectively. Then the measures $\mu^{b}, b \in\left(k_{y}^{-1}(T), g_{y}^{-1}(T)\right)$, are exactly all measures in $\mathcal{A}_{3}(T, y) \backslash \mathcal{A}_{2}(T, y)$ such that the mass is concentrated in $\alpha, b$ and $1-\alpha$ and $\int_{\mathbb{R}} q_{y}(x) \mu^{b}(d x)=T$.

Proof. Recall that if we fix the three mass points $\alpha, b, 1-\alpha$ for a measure $\mu \in \mathcal{A}_{3}(T, y) \backslash \mathcal{A}_{2}(T, y)$, then the constraints $\int_{\mathbb{R}} 1 \mu(d x)=1, \int_{\mathbb{R}} x \mu(d x)=y$ and $\int_{\mathbb{R}} q_{y}(x) \mu(d x)=T$ uniquely define the weights of a signed measure. Conditions on $b$ then guarantee that we obtain a probability measure.

Let $\mu^{b}=p_{1} \delta_{\alpha}+p_{2} \delta_{b}+\left(1-p_{1}-p_{2}\right) \delta_{1-\alpha}$ be a probability measure with $p_{1}, p_{2}, 1-p_{1}-p_{2} \in(0,1)$ and

$$
\int_{\mathbb{R}} q_{y}(x) \mu^{b}(d x)=\left(1-p_{2}\right) q(\alpha)+p_{2} q(b)-q(y)=T<q(\alpha)-q(y) .
$$

Hence $q(b)<q(\alpha)$, which implies that $b \in(\alpha, 1-\alpha)$ is necessary. Moreover, if we impose that $\mu^{b}$ satisfies $\int_{\mathbb{R}} 1 \mu^{b}(d x)=1, \int_{\mathbb{R}} x \mu^{b}(d x)=y$ and $\int_{\mathbb{R}} q_{y}(x) \mu^{b}(d x)=T$, then the weights $p_{1}, p_{2}$ and $p_{3}=1-p_{1}-p_{2}$ are given by

$$
\begin{aligned}
& p_{1}=\frac{(1-\alpha-b)(T+q(y))+(b-y) q(\alpha)-(1-\alpha-y) q(b)}{(1-2 \alpha)(q(\alpha)-q(b))}, \\
& p_{2}=\frac{q(\alpha)-(T+q(y))}{q(\alpha)-q(b)} \\
& p_{3}=\frac{(b-\alpha)(T+q(y))-(b-y) q(\alpha)-(y-\alpha) q(b)}{(1-2 \alpha)(q(\alpha)-q(b))} .
\end{aligned}
$$


Using $T<q(\alpha)-q(y)$ and $q(\alpha)>q(b)$ results in $p_{2}>0$. From

$$
(1-\alpha-b)(T+q(y)-q(\alpha))+(y-\alpha)(q(b)-q(\alpha))<0
$$

it follows that $p_{1}<1$. Note that we have $p_{2}<1$ if and only if $q(b)<T+q(y)$. Since $b \mapsto q(b)$ is continuous, symmetric around $\frac{1}{2}$ and strictly decreasing on $\left(\alpha, \frac{1}{2}\right]$ with $q(\alpha)>T+q(y)$ and $q\left(\frac{1}{2}\right)=0$, there exists a unique $\bar{b}=\bar{b}(T) \in\left(\alpha, \frac{1}{2}\right)$ with $q(\bar{b})=T+q(y)$ and $q(b)<T+q(y)$ for all $b \in(\bar{b}, 1-\bar{b})$. Thus, we restrict $b$ to the interval $(\bar{b}, 1-\bar{b})$. Since $q(y)<T+q(y)$ it follows that $y \in(\bar{b}, 1-\bar{b})$. Moreover, it holds that $p_{3}>0$ if and only if

$$
\frac{b-y}{b-\alpha} q(\alpha)+\frac{y-\alpha}{b-\alpha} q(b)<T+q(y) .
$$

For $b>y$ Inequality (5.1) can be rewritten as $g_{y}(b)<T$. From the proof of Lemma 3.1 we already know that $g_{y}(b)$ is strictly increasing in $b$. Hence, for $b>y$ Inequality (5.1) holds if and only if $b<g_{y}^{-1}(T)$. In particular, since for all $b \in\left(y, g_{y}^{-1}(T)\right)$ we have

$$
T>g_{y}(b)=\frac{b-y}{b-\alpha} q(\alpha)+\frac{y-\alpha}{b-\alpha} q(b)-q(y)>q(b)-q(y),
$$

it follows that $g_{y}^{-1}(T)<1-\bar{b}$. For $b \in(\bar{b}, y]$ we use that

$$
T+q(y)>q(b)=\frac{b-y}{b-\alpha} q(\alpha)+\frac{y-\alpha}{b-\alpha} q(b)+\frac{y-b}{b-\alpha}(q(\alpha)-q(b))>\frac{b-y}{b-\alpha} q(\alpha)+\frac{y-\alpha}{b-\alpha} q(b) .
$$

So far we have restricted $b$ to the interval $\left(\bar{b}, g_{y}^{-1}(T)\right)$. In order to obtain conditions on $b$ which guarantee that $p_{1}>0$, similar arguments as for $p_{3}>0$ apply. We have $p_{1}>0$ if and only if

$$
\frac{1-\alpha-y}{1-\alpha-b} q(b)+\frac{y-b}{1-\alpha-b} q(\alpha)<T+q(y) .
$$

Using $q(b)<T+q(y)$ yields that $(5.2)$ holds for all $b \in\left[y, g_{y}^{-1}(T)\right)$. For $b<y$ we conclude that (5.2) is satisfied if and only if $b>k_{y}^{-1}(T)$. In addition, 5.2) implies that $q(b)<T+q(y)$ for $b \in\left(k_{y}^{-1}(T), y\right)$ and hence $k_{y}^{-1}(T)>\bar{b}$. Finally, $p_{3}<1$ if and only if

$$
j(b):=(b-\alpha)(T+q(y))-(1-2 \alpha+b-y) q(\alpha)+(1-\alpha-y) q(b)<0 .
$$

The function $j$ is convex on $(0,1)$, because $j^{\prime \prime}(b)=(1-\alpha-y) q^{\prime \prime}(b)>0$ with

$$
\begin{aligned}
& j\left(k_{y}^{-1}(T)\right)=\frac{1-\alpha-y}{1-\alpha-k_{y}^{-1}(T)}(1-2 \alpha)\left(q\left(k_{y}^{-1}(T)\right)-q(\alpha)\right)<0, \\
& j\left(g_{y}^{-1}(T)\right)=(1-2 \alpha)\left(q\left(g_{y}^{-1}(T)\right)-q(\alpha)\right)<0 .
\end{aligned}
$$

Thus, for all $b \in\left(k_{y}^{-1}(T), g_{y}^{-1}(T)\right)$ the convexity of $j$ implies that $j(b)<0$. To summarize, $\mu^{b}$ is a probability measure if and only if $b \in\left(k_{y}^{-1}(T), g_{y}^{-1}(T)\right)$.

We now show that Equation (3.1) posses a unique root.

Lemma 5.2. The equation

$$
(\beta-1)(q(\alpha)-q(b))+(1-\alpha-b+b \beta-\alpha \beta) q^{\prime}(b)=0
$$

has a unique solution $b^{*} \in\left(\alpha, \frac{1}{2}\right]$ on $[\alpha, 1-\alpha]$.

Proof. For $b \in(0,1)$ let

$$
\ell(b)=(\beta-1)(q(\alpha)-q(b))+(1-\alpha-b+b \beta-\alpha \beta) q^{\prime}(b) .
$$

Observe that $\ell$ is continuous with $\ell(\alpha)=(1-2 \alpha) q^{\prime}(\alpha)<0, \ell\left(\frac{1}{2}\right)=(\beta-1) q(\alpha)>0$ if $\beta>1$ and $\ell\left(\frac{1}{2}\right)=0$ if $\beta=1$. Moreover,

$$
\ell^{\prime}(b)=(1-\alpha-b+\beta(b-\alpha)) q^{\prime \prime}(b)>0
$$

for all $b \in[\alpha, 1-\alpha]$. Hence, $\ell$ is strictly increasing on $[\alpha, 1-\alpha]$. Furthermore, there exists a unique $b^{*} \in\left(\alpha, \frac{1}{2}\right]$ such that $\ell\left(b^{*}\right)=0$. 


\section{References}

[1] S. Ankirchner, N. Kazi-Tani, M. Klein, and T. Kruse. Stopping with expectation constraints: 3 points suffice. http://hal.archives-ouvertes.fr/hal-01525439, preprint, 2017 .

[2] S. Ankirchner, M. Klein, and T. Kruse. A verification theorem for optimal stopping problems with expectation constraints. Applied Mathematics $\mathcal{E}$ Optimization, pages 1-33, 2017.

[3] S. Ankirchner, M. Klein, T. Kruse, and M. Urusov. On a certain local martingale in a general diffusion setting. https://hal .archives-ouvertes.fr/hal-01700656, preprint, 2018.

[4] E. Bayraktar and S. Yao. Dynamic programming principles for optimal stopping with expectation constraint. arXiv preprint arXiv:1708.02192, 2017.

[5] L. I. Galtchouk. Optimality of the wald sprt for processes with continuous time parameter. In A. C. Atkinson, P. Hackl, and W. G. Müller, editors, mODa 6 -Advances in ModelOriented Design and Analysis, pages 97-110, Heidelberg, 2001. Physica-Verlag HD.

[6] D. P. Kennedy. On a constrained optimal stopping problem. J. Appl. Probab., 19(3):631641, 1982.

[7] R. S. Liptser and A. N. Shiryaev. Statistics of Random Processes. I, volume 5 of Applications of Mathematics (New York). Springer-Verlag, Berlin, expanded edition, 2001.

[8] R. S. Liptser and A. N. Shiryaev. Statistics of random processes. II, volume 6 of Applications of Mathematics (New York). Springer-Verlag, Berlin, expanded edition, 2001. Applications, Translated from the 1974 Russian original by A. B. Aries, Stochastic Modelling and Applied Probability.

[9] D. I. Lisovskii. Статистическиые задачи для некоторых процессов диффузионного типа [Statistical problems for some diffusion-type processes]. PhD Thesis, 2018.

[10] D. I. Lisovskii and A. N. Shiryaev. Sequential testing of two simple hypotheses for a stationary Ornstein-Uhlenbeck process. Submitted to Theory Probab. Appl., 2018.

[11] G. Peskir and A. Shiryaev. Optimal stopping and free-boundary problems. Lectures in Mathematics ETH Zürich. Birkhäuser Verlag, Basel, 2006.

[12] A. N. Shiryaev. Optimal Stopping Rules, volume 8 of Stochastic Modelling and Applied Probability. Springer-Verlag, Berlin, 2008. Translated from the 1976 Russian second edition by A. B. Aries, reprint of the 1978 translation.

[13] A. Wald. Sequential Analysis. John Wiley \& Sons, Inc., New York; Chapman \& Hall, Ltd., London, 1947.

[14] A. Yashin. On a problem of sequential hypothesis testing. Theory of Probability \& Its Applications, 28(1):157-165, 1984. 